\title{
Coronal hole boundaries evolution at small scales
}

\section{EIS and SUMER views ${ }^{\star}$}

\author{
M. S. Madjarska ${ }^{1,2}$, Z. Huang ${ }^{1}$, J. G. Doyle ${ }^{1}$, and S. Subramanian ${ }^{1}$ \\ 1 Armagh Observatory, College Hill, Armagh BT61 9DG, N. Ireland \\ 2 UCL-Mullard Space Science Laboratory, Holmbury St Mary, Dorking, Surrey, RH5 6NT, UK \\ e-mail: madj@arm.ac.uk
}

Received 1 May 2012 / Accepted 29 June 2012

\begin{abstract}
Context. We report on the plasma properties of small-scale transient events identified in the quiet Sun, coronal holes and their boundaries.

Aims. We aim at deriving the physical characteristics of events that were identified as small-scale transient brightenings in XRT images.

Methods. We used spectroscopic co-observations from SUMER/SoHO and EIS/Hinode combined with high-cadence imaging data from XRT/Hinode. We measured Doppler shifts using single and multiple Gaussian fits of the transition region and coronal lines as well as electron densities and temperatures. We combined co-temporal imaging and spectroscopy to separate brightening expansions from plasma flows.

Results. The transient brightening events in coronal holes and their boundaries were found to be very dynamical, producing highdensity outflows at high speeds. Most of these events represent X-ray jets from pre-existing or newly emerging coronal bright points at X-ray temperatures. The average electron density of the jets is $\log _{10} N_{\mathrm{e}} \approx 8.76 \mathrm{~cm}^{-3}$ while in the flaring site it is $\log _{10} N_{\mathrm{e}} \approx 9.51 \mathrm{~cm}^{-3}$. The jet temperatures reach a maximum of $2.5 \mathrm{MK}$ but in the majority of the cases the temperatures do not exceed $1.6 \mathrm{MK}$. The footpoints of jets have maximum temperatures of $2.5 \mathrm{MK}$, though in a single event scanned a minute after the flaring the measured temperature was $12 \mathrm{MK}$. The jets are produced by multiple microflaring in the transition region and corona. Chromospheric emission was only detected in their footpoints and was only associated with downflows. The Doppler shift measurements in the quiet Sun transient brightenings confirmed that these events do not produce jet-like phenomena. The plasma flows in these phenomena remain trapped in closed loops.

Conclusions. We can conclude that the dynamic day-by-day and even hour-by-hour small-scale evolution of coronal hole boundaries reported in Paper I is indeed related to coronal bright points. The XRT observations reported in Paper II revealed that these changes are associated with the dynamic evolution of coronal bright points producing multiple jets during their lifetime until their full disappearance. We demonstrate here through spectroscopic EIS and SUMER co-observations combined with high-cadence imaging information that the co-existence of open and closed magnetic fields results in multiple energy depositions, which propel high-density plasma along open magnetic field lines. We conclude from the physical characteristics obtained in this study that X-ray jets are important candidates for the source of the slow solar wind. This, however, does not exclude the possibility that these jets are also the microstreams observed in the fast solar wind, as recently suggested.
\end{abstract}

Key words. Sun: corona - Sun: transition region - line: profiles - methods: observational

\section{Introduction}

Coronal holes (CHs) are regions on the Sun dominated by open magnetic fields. They are seen with reduced emission in spectral lines formed at coronal temperatures and are identified as the source regions of the fast solar wind with velocities of $\sim 800 \mathrm{~km} \mathrm{~s}^{-1}$ (Krieger et al. 1973). The CHs form in both polar and equatorial regions. The latter are often connected with the polar $\mathrm{CHs}$ with a channel of open magnetic field and are called equatorial extensions of polar coronal holes (EECHs). These exhibit a more rigid rotation (Timothy et al. 1975) with respect to the typical differential rotation at photospheric levels. Therefore, it is believed that interchange magnetic reconnection happens between the open (coronal hole's) and closed (quiet Sun) magnetic field lines. This was suggested to play an important role in the generation of the slow solar wind flow

* Figures A.1, A.2, and movies are available in electronic form at http://www. aanda.org
(Wang et al. 1998b; Woo et al. 2004). Evidence of dynamic processes taking place at coronal hole boundary $(\mathrm{CHB})$ regions was first provided from spectroscopic observations by Madjarska et al. (2004). It has been found that events described by nonGaussian profiles of transition region spectral lines, e.g. in N IV $765.15 \AA$ and Ne VIII $770.43 \AA$, were abundant along the boundary of an EECH. Similar results were found at a polar coronal hole boundary by Doyle et al. (2006) in O vi $1031.93 \AA$. More details on the background of $\mathrm{CHs}$ and coronal hole boundaries can be found in Madjarska \& Wiegelmann (2009, hereafter Paper I) and Subramanian et al. (2010, hereafter Paper II).

Madjarska \& Wiegelmann (2009) showed that although isolated equatorial $\mathrm{CHs}$ and $\mathrm{EECHs}$ maintain their general shape during several solar rotations, a closer look at their day-by-day and hour-by-hour evolution demonstrates significant dynamics. Using the Extreme-ultraviolet Imaging Telescope (EIT)/SoHO $195 \AA$ and TRACE $171 \AA$ observations, they found that evolution of small-scale loops, i.e. coronal bright 
points (BPs), led to small-scale changes of the $\mathrm{CH}$ boundaries. The disappearance of BPs was associated with an expansion of the $\mathrm{CHs}$ while the appearance of BPs was followed by contraction of the CHs.

Since the launch of the Hinode satellite, coronal holes are seen in observations from the X-ray Telescope (XRT, Golub et al. 2007) as highly structured and dynamically evolving at small scales as never before. We analysed XRT images taken with the Al_poly filter studying transient brightenings in $\mathrm{CH}$, $\mathrm{CHB}$ and quiet-Sun regions (Paper II). We found that $70 \%$ of the brightening events in $\mathrm{CHs}$ and at $\mathrm{CH}$ boundaries appear as loop expulsions and/or collimated outflows while only $30 \%$ of the events in the quiet Sun showed possible flows/outflows. We also found that the ejected plasma during outflow events always originated from pre-existing or newly emerging coronal bright points at X-ray temperatures. Madjarska (2011) studied in great detail an X-ray jet based on unique imager and spectrometer co-observations. The author found that the outflow is composed both of BP loop expulsion and collimated outflow with several energy depositions during the lifetime of the event. Hot plasma was seen to rise earlier than the cooler plasma. The spectroscopic study revealed two main flows for the jet, a fast outflow with Doppler velocities around $300 \mathrm{~km} \mathrm{~s}^{-1}$ and a downflow guided by closed magnetic field lines with Doppler velocities of 25 to $50 \mathrm{~km} \mathrm{~s}^{-1}$ at transition region temperatures and $150 \mathrm{~km} \mathrm{~s}^{-1}$ at coronal temperatures. Madjarska (2011) also established that multiple energy depositions happened during the jet.

$\mathrm{X}$-ray jets were first discovered in observations by the Soft X-ray Telescope (SXT) onboard Yohkoh. They are associated with BPs (Shibata et al. 1992, 1994; Doschek et al. 2010; Wang et al. 1998a; Subramanian et al. 2010). In a statistical study, Shimojo et al. (1996) concluded that they have an average length of a few $10^{4}$ to $4 \times 10^{5} \mathrm{~km}$, widths of $5 \times 10^{3}$ to $10^{5} \mathrm{~km}$, velocities of 10 to $1000 \mathrm{~km} \mathrm{~s}^{-1}$ with an average velocity of $200 \mathrm{~km} \mathrm{~s}^{-1}$ and lifetime of up to $10 \mathrm{~h}$. Although $68 \%$ of X-ray jets were discovered in or near active regions, they can also be seen in the quiet Sun and CHs. Furthermore, Shimojo \& Shibata (2000) analysed the physical parameters of $16 \mathrm{X}$-ray jets using imaging data from the soft X-ray telescope onboard the Yohkoh satellite. They derived the temperature of the jets and their flaring footpoint as $3-8 \mathrm{MK}$. The electron density of the jets was found to be $0.7-4 \times 10^{9} \mathrm{~cm}^{-3}$ while for the flaring site $2.4-10.0 \times 10^{9} \mathrm{~cm}^{-3}$. These events are also observed in the ultraviolet (Alexander \& Fletcher 1999; Ko et al. 2005; Madjarska 2011) and white-light (Paraschiv et al. 2010). Paraschiv et al. (2010) analysed over 10000 events observed in white-light by STEREO/SECCHICOR1, and found that little more than $70 \%$ of the white-light jets were associated with EUV jets. Usually, X-ray jets are associated with small-scale flares in their footpoints (Shibata et al. 1992). Hence, magnetic reconnection is suggested as their formation mechanism (Shibata et al. 1992; Yokoyama \& Shibata 1995; Shimojo \& Shibata 2000; Moreno-Insertis et al. 2008; Pariat et al. 2010).

The occurrence of non-Gaussian profiles in spectral lines from ions formed at transition region temperatures was named an "explosive event" (EEs) with no imaging information of what phenomenon actually produces these line profiles. These events were studied for the past three decades using observations obtained first by the Naval Research Laboratory High Resolution Telescope and Spectrometer (HRTS) and, later, the Solar Ultraviolet Measurements of Emitted Radiation (SUMER) spectrograph onboard the Solar and Heliospheric Observatory (SoHO). Explosive events were found to have an average lifetime of 200 s and a size of $4^{\prime \prime}-5^{\prime \prime}$ determined by the width of
Table 1. The spectral lines registered with EIS.

\begin{tabular}{|c|c|c|}
\hline Ion & Wavelength $(\AA)$ & $\log T_{\max }(\mathrm{K})$ \\
\hline $\mathrm{He}_{\text {II }}^{*}$ & $256.32 \mathrm{~b}$ & 4.7 \\
\hline $\mathrm{O} \mathrm{v}^{*}$ & 192.90 & 5.4 \\
\hline $\mathrm{O} \mathrm{vI}^{*}$ & 184.12 & 5.5 \\
\hline Mg vI & $270.39 b$ & 5.7 \\
\hline \multirow[t]{2}{*}{ Mg VII } & 278.39 & 5.8 \\
\hline & 280.75 & 5.8 \\
\hline Si VII* & 275.35 & 5.8 \\
\hline Fe viII ${ }^{*}$ & 185.21 & 5.8 \\
\hline $\mathrm{Fe}^{*}$ & 184.54 & 6.0 \\
\hline $\mathrm{Fe} \mathrm{XI}$ & 188.23 & 6.1 \\
\hline Si $x$ & 258.37 & 6.1 \\
\hline Si x* & 261.04 & 6.1 \\
\hline Fe XII* & 195.12 & 6.1 \\
\hline \multirow[t]{2}{*}{ Fe XIII* } & 202.04 & 6.2 \\
\hline & 203.82 & 6.2 \\
\hline Fe $\mathrm{XIV}^{*}$ & $274.20 \mathrm{~b}$ & 6.3 \\
\hline $\mathrm{Fe} \mathrm{xv}^{*}$ & 284.16 & 6.3 \\
\hline Fe XVI & 262.98 & 6.4 \\
\hline $\operatorname{Ar} x v$ & 293.69 & 6.6 \\
\hline Fe XXIII & 263.76 & 7.1 \\
\hline
\end{tabular}

Notes. The comment "b" means that the spectral line is blended. The spectral lines with an asterisk were taken during the January 2009 observations.

the blue- and red-shifted emission along a spectrometer slit. The EEs show Doppler velocities of up to $200 \mathrm{~km} \mathrm{~s}^{-1}$ that are predominantly blue-shifted and were found at the boundaries of the super-granulation cells. The main characteristics of EES were studied by Brueckner \& Bartoe (1983); Dere et al. (1989); Porter \& Dere (1991); Madjarska \& Doyle (2002, 2003, and the references therein). It has been suggested that EEs are the spectroscopic signature of magnetic reconnection happening in the transition region (Dere 1994; Innes et al. 1997). In recent years, simultaneous imaging and spectroscopic observations helped to shed more light on the nature of this phenomenon. The EEs were found to be produced by a siphon flow in a small-scale loop (Teriaca et al. 2004). They also result from the up- and downflows in a surge (Madjarska et al. 2009).

Here, we present a follow-up study on coronal hole boundary evolution, namely a spectroscopic analysis of some of the brightening events identified in the XRT images from November 2007 and January 2009 studied in Paper II. In Sect. 2 we describe the observations. The data analysis and results are given in Sect. 3 . The discussion and conclusions are outlined in Sect. 4.

\section{Observations}

The observations used for the present study were taken in November 2007 during a dedicated multi-instrument Hinode and SoHO campaign with the SUMER/SoHO and Extremeultraviolet Imaging Telescope (EIS)/Hinode. An equatorial coronal hole was tracked from the east to the west limb during four days. Details on the registered EIS and SUMER spectral lines can be found in Tables 1 and 2, respectively. Details on the observing times and pointing of the SUMER and EIS instruments are given in Tables 3 and 4 . We also analysed data taken in the quiet Sun in January 2009 (all information on these data is also included in Tables 1-4). The field-of-view (FOV) of XRT, EIS and SUMER are shown in Figs. 1 and 2. The SUMER and EIS data reduction and alignment were performed as described in Madjarska (2011). 
Table 2. The SUMER spectral lines.

\begin{tabular}{|c|c|c|}
\hline Ion & Wavelength $(\AA)$ & $\log T_{\max }(\mathrm{K})$ \\
\hline $\mathrm{Nv}$ & 1238.82 & 5.3 \\
\hline \multirow[t]{2}{*}{$\mathrm{C}_{\mathrm{I}}$} & 1248.00 & 4.0 \\
\hline & 1248.88 & \\
\hline $\mathrm{C}_{\mathrm{I}}$ & 1249.00 & 4.0 \\
\hline $\mathrm{O} \mathrm{IV} / 2$ & $1249.24 b$ & 5.2 \\
\hline $\mathrm{Si} \mathrm{x} / 2$ & $1249.30 \mathrm{~b}$ & 6.1 \\
\hline $\mathrm{C}_{\mathrm{I}}$ & 1249.41 & 4.0 \\
\hline $\operatorname{Mg} x / 2$ & 1249.90 & 6.1 \\
\hline $\mathrm{O}$ IV $/ 2$ & $1250.25 b$ & 5.2 \\
\hline Si II & 1250.09 & 4.1 \\
\hline Si II & 1250.41 & 4.1 \\
\hline $\mathrm{C}_{\mathrm{I}}$ & $1250.42 b$ & 4.0 \\
\hline $\mathrm{S}_{\text {II }}$ & 1250.58 & 4.2 \\
\hline $\mathrm{Si}$ II & 1251.16 & 4.1 \\
\hline $\mathrm{C}_{\mathrm{I}}$ & $1251.17 \mathrm{~b}$ & 4.0 \\
\hline \multirow[t]{2}{*}{$\mathrm{O}$ IV/2 } & 1251.70 & 5.2 \\
\hline & 1251.78 & \\
\hline $\mathrm{Si}$ I & 1256.49 & 4.0 \\
\hline $\mathrm{Si}$ I & 1258.78 & 4.1 \\
\hline $\mathrm{S}_{\text {II }}$ & $1259.53 b$ & 4.2 \\
\hline $\mathrm{O} v / 2$ & 1259.54 & 5.4 \\
\hline Si II & 1260.44 & 4.1 \\
\hline Si IV & 1393.78 & 4.9 \\
\hline
\end{tabular}

Notes. The expression "/2" means that the spectral line was observed in second order. The comment "b" means that the spectral line is blended with a close-by line.

\subsection{EIS}

The EIS (Culhane et al. 2007a) study was specially designed to provide the best possible coverage of spectral lines with formation temperatures $\left(\log T_{\max }\right)$ from $4.7 \mathrm{~K}$ to $7.1 \mathrm{~K}$. First, a raster of $120^{\prime \prime} \times 512^{\prime \prime}$ was obtained, followed by small rasters of $24^{\prime \prime} \times 512^{\prime \prime}$. The $2^{\prime \prime}$ slit with an exposure time of $60 \mathrm{~s}$ was used. During the January 2009 run the rasters had a size of $70^{\prime \prime} \times 248^{\prime \prime}$ and were taken with a $40 \mathrm{~s}$ exposure time. The Doppler shift maps were derived from a single Gaussian fit with the reference line obtained as an average over the entire raster after all instrumental effects were removed. The Doppler velocities measured here should be considered as indicative only of the range of the Doppler shift rather than absolute values because EIS does not have an absolute calibration, although for some type of observations Doppler measurements close to their absolute values can be obtained (Young et al. 2012).

\subsection{SUMER}

The SUMER (Wilhelm et al. 1995; Lemaire et al. 1997) observations were made with a slit size of $1^{\prime \prime} \times 300^{\prime \prime}$. The detector B was exposed for $60 \mathrm{~s}$ while the spectrometer was observing in a sit-and-stare mode. The time sequence was followed by a raster with a size of $60^{\prime \prime} \times 300^{\prime \prime}$ made only in the O v $629.77 \AA$ and Si I $1256.49 \AA$ lines and a $30 \mathrm{~s}$ exposure time. The observations were compensated for the solar rotation. Five spectral windows were transferred to the ground each with a size of 50 spectral pixels $\times 300$ spatial pixels. During the November 9 observations the detector read-outs were shifted by about 20 spectral pixels. As a result, the $\mathrm{O} v$ and $\mathrm{N} v$ lines have part of their blue wing cut, which limited the line blueshift measurements to only $92 \mathrm{~km} \mathrm{~s}^{-1}$. From all lines only O v $629.77 \AA$ (in second order of reflection) was taken on the bare part of the detector.

\section{Data analysis, results and discussion}

The first step of our study was to align the EIS rasters and SUMER sit-and-stare data with the XRT images analysed in aper II (see Figs. 1 and 2). Next, we located all brightenings identified in the XRT images (Paper II) on the EIS raster images. The brightening events were grouped as in Paper II, i.e., we inspected the image sequence of each dataset with the identified brightenings overplotted at corresponding times (see Figs. A.1 and A.2). Clusters of bright pixels identified next to each other and evolving at the same or at very close times were selected as one event. In Figs. 3 and 4, the brightenings are overplotted on the $\mathrm{CH}$ and QS images, respectively. Once each group of brightenings was unified into an individual event, each such event was given a number and was marked as a quiet-Sun, a CHB or a $\mathrm{CH}$ event. A coronal hole boundary is defined as the region of $\pm 15^{\prime \prime}$ on both sides of the contour line outlining the coronal hole boundary as determined on the XRT images in Paper II. Part of the events registered in November 2007 were also recorded by the SUMER spectrometer in the spectral lines shown in Table 2. We produced image sequences of all data taken at similar times to obtain a visual picture of the event evolution. The combination of spectroscopic and imager data presented in this manner is the best existing way to obtain a full description of an observed phenomenon using the presently existing solar instrumentation. Examples of this kind of "movies" are given in the online material (see Figs. A.1 and A.2) ${ }^{1}$. We then derived the physical plasma parameters of each event, i.e. their structural evolution in time, Doppler shifts, temperatures, and densities. Because of the way slit spectrometers operate and because of the random occurrence of transient brightenings, we were able to "catch" the physical parameter evolution in various parts of these events and at various stages of the event's development. Unfortunately, the event best observed by all instruments remains the one discussed in detail by Madjarska (2011).

In Figs. 1 and 2 we present the XRT images studied in Paper II that were used to automatically identify transient brightenings. From the inspection of the composed image animations, we were able to visually determine the spectroscopic signature of each brightening. The analysis is not trivial and very timeconsuming because it requires considering of each type of observation, i.e. a rastering and a sit-and-stare, the exposure duration, contaminations from line-of-sight contributions, and last but not least, various instrumental effects that are specific for each instrument. The number of QS events that were found in the November 2007 data is small in comparison with the number of $\mathrm{CH}$ events because of the limited FOV of the instruments. To increase the number of analysed QS events, we also used the two QS datasets taken on 2009 January 10 and 13. The EIS observations for this run were performed with a different program, i.e. only larger rasters were taken. Nevertheless, these data provide the same quality information as the November 2007 run. In Figs. 3 and 4, the EIS FOVs (only the big rasters for November 2007) are shown for all observations. The brightening pixels as identified through an automatic procedure (Paper II) in XRT Alpoly images are overplotted with symbols. The solid and dashed (only in Fig. 3) line boxes correspond to the identification during the big and small rasters, respectively. The coronal hole boundaries are defined to match the boundaries considered in Paper II. All physical parameters are given in Tables 5-7 are discussed in detail in the next sections.

\footnotetext{
1 Movies for each dataset in 2007 and 2009 can be found at http://star.arm.ac.uk/highlights/2012/599-media/
} 
Table 3. Description of the EIS observations used in this study.

\begin{tabular}{|c|c|c|c|c|c|}
\hline \multirow[t]{3}{*}{ Date } & \multirow{3}{*}{$\begin{array}{c}\text { Observing period } \\
\text { (UT) }\end{array}$} & \multicolumn{3}{|c|}{ Heliospheric coordinates $(\min -\max )$} & \multirow{3}{*}{ Number of rasters } \\
\hline & & \multicolumn{2}{|c|}{ Solar $X(\operatorname{arcsec})$} & \multirow[t]{2}{*}{ Solar $Y(\operatorname{arcsec})$} & \\
\hline & & Big raster & Small raster & & \\
\hline 2007-Nov-09 & $06: 35 \rightarrow 14: 50$ & $-545.2 \rightarrow-425.2$ & $-499.3 \rightarrow-475.3$ & $-421.1 \rightarrow-24.1$ & 1 big, 29 small \\
\hline 2007-Nov-12 & $01: 17 \rightarrow 10: 43$ & $-40.8 \rightarrow 79.2$ & $8.2 \rightarrow 32.2$ & $-432.0 \rightarrow-35.0$ & $1 \mathrm{big}, 35$ small \\
\hline 2007-Nov-14 & $00: 20 \rightarrow 10: 48$ & $208.9 \rightarrow 328.9$ & $261.0 \rightarrow 285.0$ & $-382.6 \rightarrow 14.4$ & $1 \mathrm{big}, 40$ small \\
\hline 2007-Nov-16 & $18: 07 \rightarrow 23: 47$ & $729.1 \rightarrow 849.1$ & $784.3 \rightarrow 808.3$ & $-341.3 \rightarrow 55.7$ & 1 big, 22 small \\
\hline 2009-Jan-10 & $11: 30 \rightarrow 17: 27$ & -100.0 & $\rightarrow-30.0$ & $-146.7 \rightarrow 101.3$ & 14 \\
\hline 2009-Jan-13 & $11: 22 \rightarrow 17: 41$ & -101.2 & $\rightarrow-31.2$ & $-147.0 \rightarrow 101.0$ & 15 \\
\hline
\end{tabular}

Table 4. Description of the SUMER observations used in this study.

\begin{tabular}{|c|c|c|c|c|}
\hline \multirow[t]{2}{*}{ Date } & \multicolumn{2}{|c|}{ Observing period (UT) } & \multicolumn{2}{|c|}{ Heliospheric coordinates } \\
\hline & Sit-and-stare & Raster & Solar $X(\operatorname{arcsec})$ & Solar $Y(\operatorname{arcsec})$ \\
\hline 2007-Nov-09 & $\begin{array}{l}07: 01 \rightarrow 08: 41 \\
11: 01 \rightarrow 12: 42\end{array}$ & $\begin{array}{l}08: 43 \rightarrow 08: 58 \\
12: 43 \rightarrow 12: 58\end{array}$ & -501.6 & $-339.1 \rightarrow-39.1$ \\
\hline 2007-Nov-12 & $\begin{aligned} 01: 35 & \rightarrow 03: 16 \\
05: 34 & \rightarrow 07: 14\end{aligned}$ & $\begin{array}{l}03: 17 \rightarrow 03: 31 \\
07: 16 \rightarrow 07: 31\end{array}$ & -5.6 & $-345.0 \rightarrow-45.0$ \\
\hline 2007-Nov-14 & $\begin{array}{l}01: 01 \rightarrow 02: 41 \\
02: 58 \rightarrow 04: 39 \\
07: 01 \rightarrow 08: 42\end{array}$ & $\begin{aligned} 02: 43 \rightarrow 02: 56 \\
04: 40 \rightarrow 04: 55 \\
08: 43 \rightarrow 11: 02\end{aligned}$ & 245.2 & $-300.6 \rightarrow-0.6$ \\
\hline 2007-Nov-16 & $\begin{aligned} 18: 01 & \rightarrow 19: 41 \\
22: 16 & \rightarrow 23: 41\end{aligned}$ & $\begin{aligned} 19: 42 & \rightarrow 19: 57 \\
23: 43 & \rightarrow 23: 58\end{aligned}$ & 750.5 & $-259.3 \rightarrow 40.7$ \\
\hline
\end{tabular}
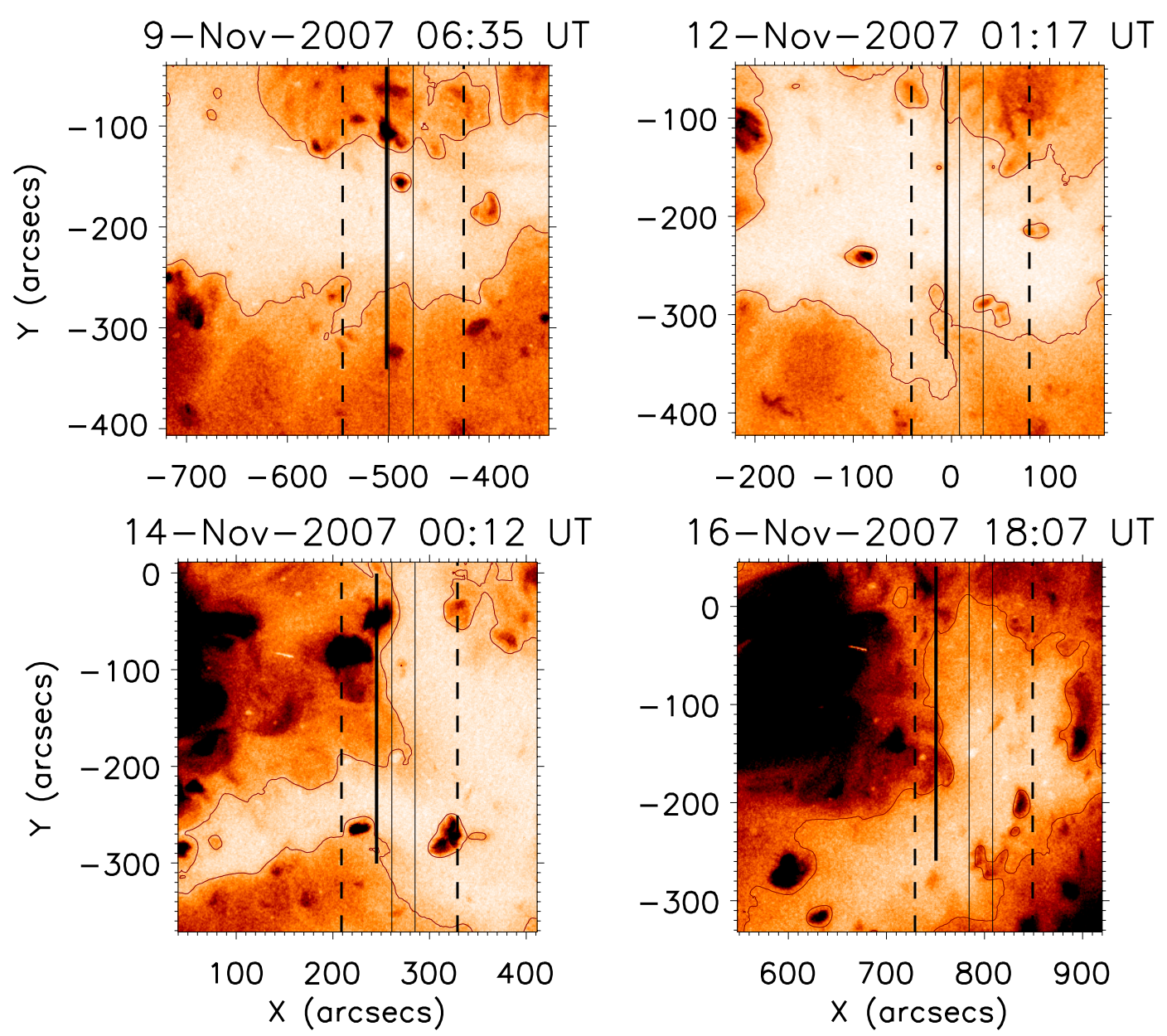

Fig. 1. XRT images of the four days of coronal hole observations. The dashed lines show the large EIS raster field-of-view while the thin solid lines denote the small raster FOV. The SUMER slit-and-stare position is shown with a thick solid line. Contours of XRT images outline the coronal holes as defined in Paper II. 
M. S. Madjarska et al.: Coronal hole boundaries at small scales. III.
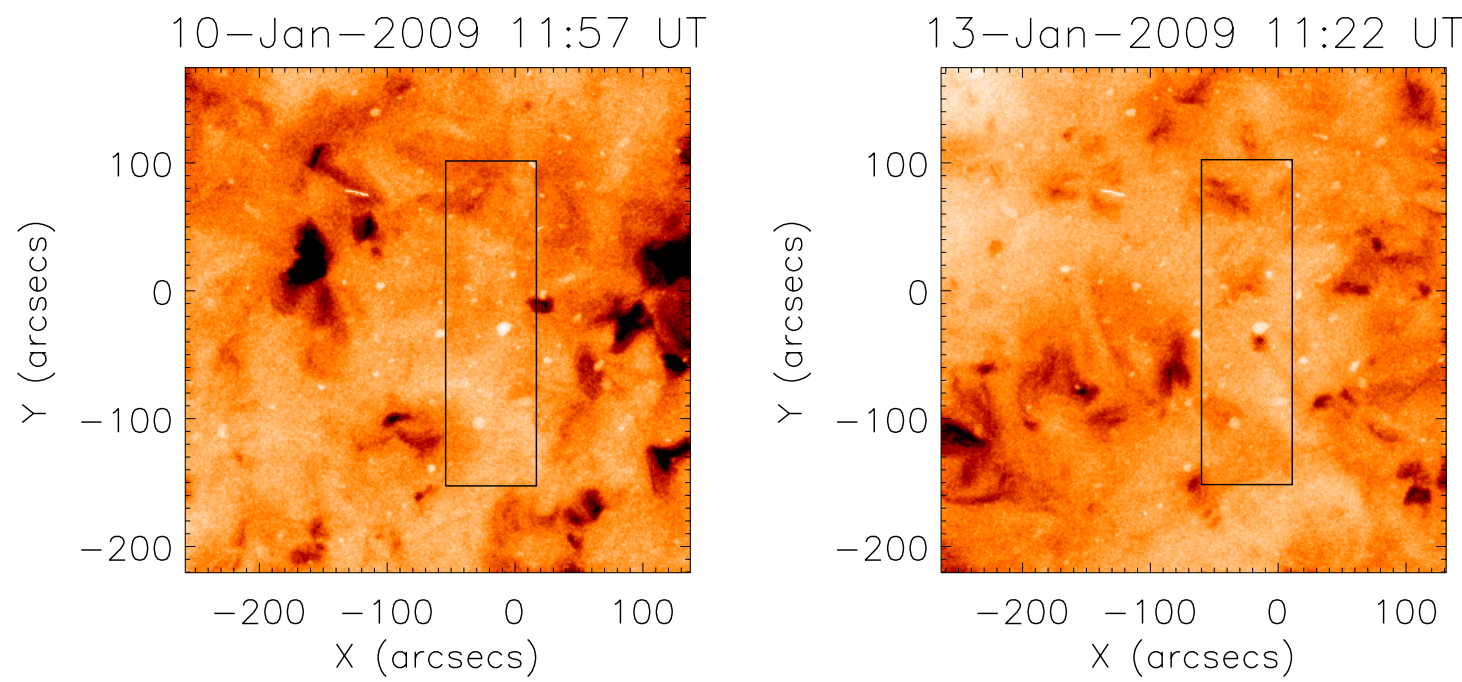

Fig. 2. XRT images taken on 2009 January 10 (left) and 13 (right) on the quiet Sun with the field-of-view of the EIS rasters overplotted.
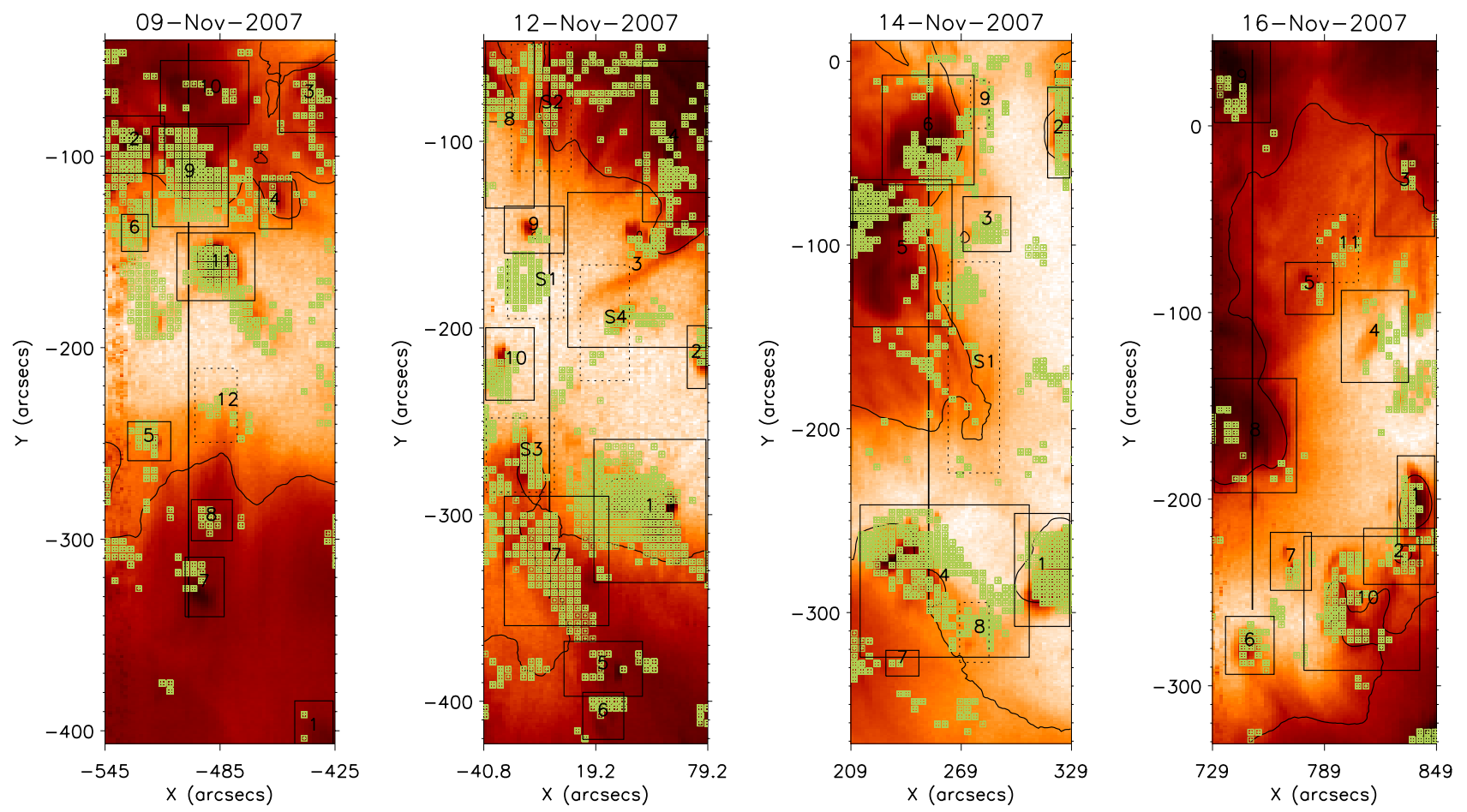

Fig. 3. EIS large rasters with the identified events overplotted with solid line boxes. The dashed line boxes indicate the events identified in the small rasters. The overplotted symbols show the pixels identified with a brightening enhancement in the XRT data from Paper II.

\subsection{Quiet Sun brightenings}

\subsubsection{Structure and dynamics}

The quiet-Sun brightenings (15 in total) all evolve starting with a brightening in an area of a few pixels (microflaring). The evolution proceeds in several patterns that seem to depend on the size of the phenomenon: (1) the initial brightening can be seen spreading along a loop system or just a single loop (observed at the EIS and XRT spatial resolution); (2) it may evolve from a point-like brightening ( $\sim 3-4$ pixels) that may enlarge slightly and then quickly (1-2 min) fade away; (3) microflaring occurs in pre-exiting BPs, which triggers a brightening increase over the whole or large parts of the pre-exiting BP. In the latter case, loops are often seen to expand from the BP and then shrink back to their original size. In many cases no feature is present at X-ray temperatures prior to the transient brightening. That clearly indicates that after energy deposition(s), the plasma was heated to $\mathrm{X}$-ray temperatures and/or then ejected along the magnetic fieldlines of pre-existing or newly formed loops.

To investigate the plasma properties and dynamics of the transient brightenings we analysed EIS Doppler shift maps and intensity images in spectral lines covering a wide temperature range (see Tables 1 and 2) together with co-temporally taken $\mathrm{X}$-ray images. This way of analysing transient events permits to separate a brightening expansion from a plasma flow. The online material provides some of the observational material used in the present study (Figs. A.1 and A.2). In addition, we present three events in Figs. 5-7 that are typical examples of QS transient phenomena. One should bear in mind that the Doppler velocity images were produced from a single Gaussian fit and, therefore, do 
Table 5. Plasma parameters of the brightening events observed in the quiet Sun.

\begin{tabular}{|c|c|c|c|c|c|c|}
\hline \multirow{2}{*}{$\begin{array}{c}\text { Date and } \\
\text { event number }\end{array}$} & \multicolumn{2}{|c|}{ Doppler shifts $\left(\mathrm{km} \mathrm{s}^{-1}\right)$} & \multirow{2}{*}{$\begin{array}{c}\text { Density } \\
\log _{10} N_{\mathrm{e}}\left(\mathrm{cm}^{-3}\right) \\
\end{array}$} & \multirow{2}{*}{$\begin{array}{l}\text { Line } \\
\text { at } T_{\max }\end{array}$} & \multirow{2}{*}{$\begin{array}{l}\text { Flow } \\
\text { outflow }\end{array}$} & \multirow{2}{*}{$\begin{array}{l}\text { Occurrence rate } \\
\text { and appearance }\end{array}$} \\
\hline & Blue & Red & & & & \\
\hline \multicolumn{7}{|l|}{$09 / 11 / 07$} \\
\hline 1 & -5 & - & 8.61 & Fe XIII & $\mathrm{Y}^{\text {later }}$ & $>2$; unresolved brightening \\
\hline 7 & - & 2.5 & 9.01 & Fe $x v$ & $\mathrm{~N}$ & $>1$, BP with evolving loops \\
\hline 8 & -3 & 3.5 & 8.58 & $\mathrm{Fe}$ XIII & $\mathrm{N}$ & $>1$; unresolved brightening \\
\hline 10 & -3 & 1.5 & 8.84 & $\mathrm{Fe} \mathrm{xv}$ & $\mathrm{N}$ & $>2$; brightening in QS loops \\
\hline \multicolumn{7}{|l|}{$12 / 11 / 07$} \\
\hline 4 & -2 & 2 & 8.92 & Fe xIII & $\mathrm{N}$ & numerous identified events in a large BP (small AR) \\
\hline \multicolumn{7}{|l|}{$14 / 11 / 07$} \\
\hline 7 & -2 & - & 8.65 & Fe xIII & $\mathrm{N}$ & $>2$; point-like brightening \\
\hline \multicolumn{7}{|l|}{$16 / 11 / 07$} \\
\hline 9 & -6 & 6 & 8.76 & $\mathrm{Fe}$ XIII & Y & $>2$; large $\mathrm{BP}$ \\
\hline \multicolumn{7}{|l|}{$10 / 01 / 09$} \\
\hline 1 & $-19 /-12$ & 80 & 9.38 & $\mathrm{Fe} x \mathrm{x}$ & $\mathrm{N}$ & 1 ; brightenings in a loop \\
\hline 2 & -2 & - & 8.78 & $\mathrm{Fe}$ xIII & $\mathrm{N}$ & 2 ; brightenings in a compact $\mathrm{BP}$ \\
\hline 3 & $-8 /-5$ & 7 & 8.70 & Fe XIII & $\mathrm{Y}$ & $>2$; brightenings in a small-scale loop \\
\hline 4 & -2 & 5 & 8.85 & $\mathrm{Fe}$ XIII & $\mathrm{N}$ & 1 ; brightenings in part of a larger loop structure \\
\hline \multicolumn{7}{|r|}{ 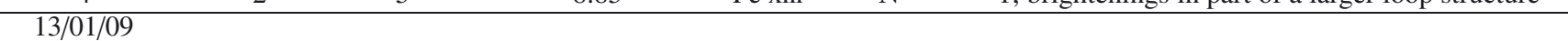 } \\
\hline 1 & -3 & - & 9.25 & $\mathrm{Fe} x \mathrm{xv}$ & $\mathrm{Y}$ & 5; compact long-lived $\mathrm{BP}$ \\
\hline 2 & -3 & - & 8.90 & $\mathrm{Fe}$ XIII & $\mathrm{Y}$ & 1 ; brightenings in a small-scale loop \\
\hline 3 & -2 & _ & 8.73 & $\mathrm{Fe}$ XIII & $\mathrm{N}$ & 1; brightenings in a small-scale loop \\
\hline 4 & -6 & 8 & 9.08 & Fe xIII & $\mathrm{N}$ & $>2 ;$ small BP \\
\hline
\end{tabular}

Notes. "Later" indicates that the outflow was seen after the EIS rastering was completed and was only registered in XRT.
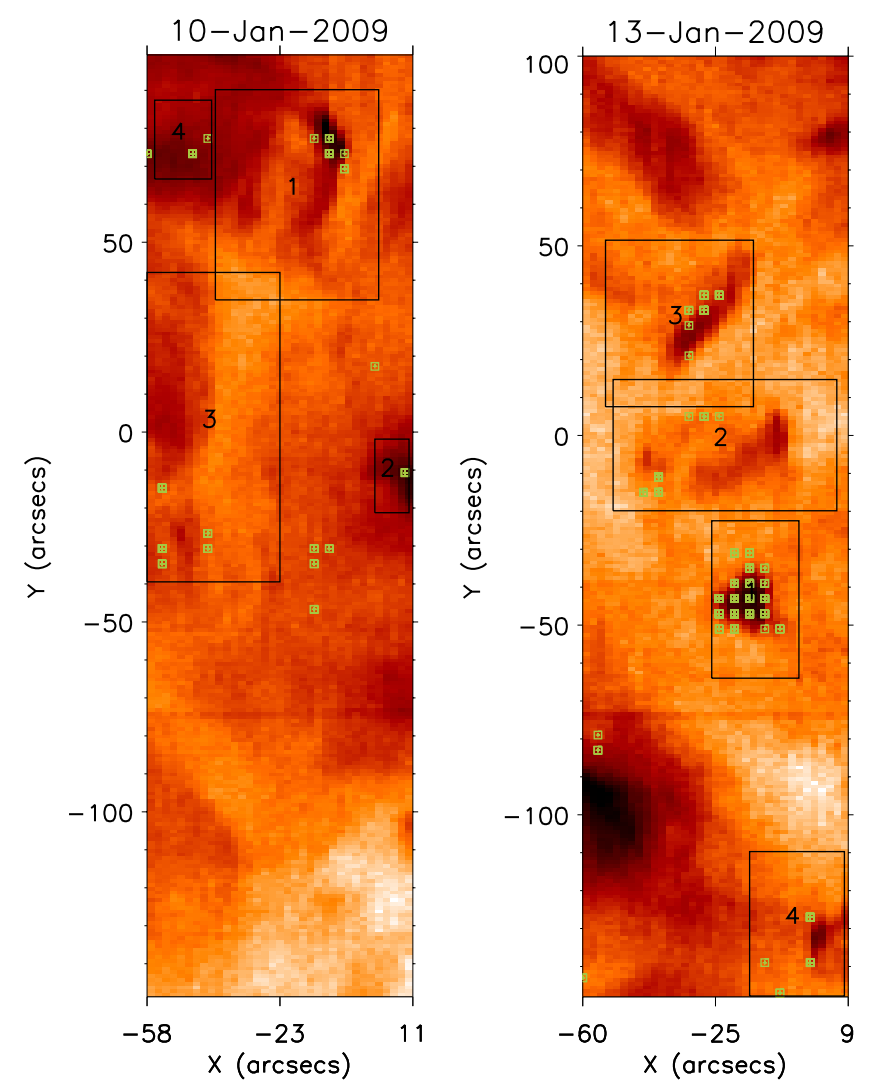

Fig. 4. EIS rasters on 2009 January 10 (left) and 13 (right). The overplotted symbols show the position of the identified brightenings.

not reflect the exact pattern of the plasma flows. These Doppler images, however, give a good indication of the dominant flows during the rastering of the events.

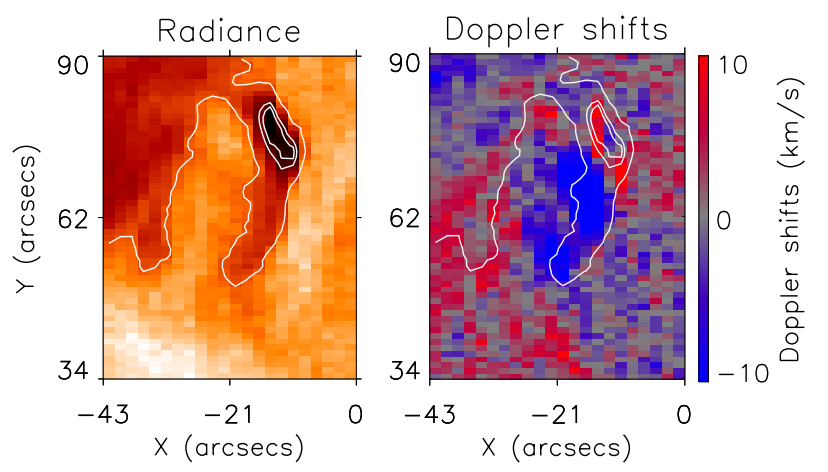

Fig. 5. Event No. 1 on January 10. The raster was taken in the Fe XII $195 \AA$ line between 12:35 UT and 12:59 UT. The left and right panels represent the Fe XII $195 \AA$ intensity and Doppler shift images, respectively. The intensity image is plotted in negative logarithmic scale.

The event in Fig. 5 started with a brightening in a single pixel, as seen in the XRT image at 12:20 UT (Fig. A.1). The brightening spread over more pixels until a loop structure is clearly visible in the XRT image taken 6 min later (Fig. 5). During the EIS scan starting at 12:35 UT, the feature shows clear red- and blue-shift patterns. The red-shift coincides with the energy deposition site while the blue-shift appears along a loop structure originating from the energy deposition site. Several more brightenings re-occurred in the same structure but they were not identified as brightenings in the XRT data in Paper II because they did not meet the selection criteria adopted there. The flows remain trapped in closed loops.

In Fig. 6 we show a feature that is a typical example of a coronal bright point (BP) with a diameter of less than $20^{\prime \prime}$. The event was scanned by EIS during several rasters and the first feature to notice is that the general blue-red Doppler-shift pattern of this phenomenon remains very similar during the entire lifetime of the BP, i.e. half of the brightening shows 
M. S. Madjarska et al.: Coronal hole boundaries at small scales. III.

Table 6. As Table 5, presenting the plasma properties of the events observed inside coronal holes and at coronal hole boundaries.

\begin{tabular}{|c|c|c|c|c|c|c|}
\hline $\begin{array}{c}\text { Date and } \\
\text { event number }\end{array}$ & \multicolumn{2}{|c|}{ Doppler shifts $\left(\mathrm{km} \mathrm{s}^{-1}\right)$} & $\begin{array}{c}\text { Density } \\
\log _{10} N_{\mathrm{e}}\left(\mathrm{cm}^{-3}\right) \\
\end{array}$ & \multirow[t]{2}{*}{$\begin{array}{c}\text { Line } \\
\text { at } T_{\max }\end{array}$} & $\begin{array}{c}\text { Flow/ } \\
\text { outflow }\end{array}$ & $\begin{array}{c}\text { Occurrence rate, } \\
\text { appearance, Phase }\end{array}$ \\
\hline $09 / 11 / 07$ & & & & & & \\
\hline 2 & $-140 /-8$ & 4.8 & 9.23 & $\mathrm{Fe} x \mathrm{v}$ & $\mathrm{Y}$ & $>2$; compact BP; post brightening phase \\
\hline 3 & -5 & 4 & 8.98 & Fe XIII & $\mathrm{N}$ & $>2$; pre-activity phase, only brightening, no jet yet; $\mathrm{BP}$ \\
\hline 4 & -2 & 2 & 8.96 & $\mathrm{Fe}$ XIII & $\mathrm{Y}^{\text {later }}$ & 2; point-like brightening, quiet phase \\
\hline 5 & - & $3 / 77$ & 9.32 & $\mathrm{Fe}$ XIII & $\mathrm{Y}$ & 2,1 large; point-like brightening \\
\hline 6 & -5 & $3 / 180$ & 9.21 & $\mathrm{Fe}$ XIII & $\mathrm{Y}$ & $>1,1$ large, flow in a small-scale loop \\
\hline 9 & $-199 /-3$ & 15 & 9.24 & $\mathrm{Fe} x \mathrm{x}$ & $\mathrm{Y}$ & $>3,2$ large; large $\mathrm{BP}$ \\
\hline 11 & $-160 /-9$ & 12 & 9.48 & $\mathrm{Fe} x \mathrm{v}$ & $\mathrm{Y}$ & $>3$; long-lived BP \\
\hline 12 & $-160 /-8$ & 2 & 8.60 & Fe XIII & $\mathrm{Y}$ & 1 ; jet from point-like brightening \\
\hline \multicolumn{7}{|l|}{$12 / 11 / 07$} \\
\hline 1 & $-170 /-20$ & $15 / 76$ & 9.37 & Fe xv & Y & $>3$; large jets, slit crosses the whole event \\
\hline 2 & - & 2.6 & 9.26 & Fe XIII & $\mathrm{N}$ & $>3$ later; quiet phase \\
\hline 3 & -170 & 7 & 8.76 & $\mathrm{Fe}$ XIII & $\mathrm{Y}$ & 1 large but faint, slit crosses the jet \\
\hline 5 & - & 8 & 8.85 & $\mathrm{Fe} \mathrm{xv}$ & $\mathrm{N}$ & $>2$; point-like brightening \\
\hline 6 & $-10 /-150 /$ & - & 8.66 & Fe xv & $\mathrm{Y}$ & 2 ; slit crosses only the outflow \\
\hline 7 & $-140 /-20$ & - & 8.74 & Fe XIII & $\mathrm{Y}$ & 1 large; slit crosses only the jet \\
\hline 8 & - & 22 & 8.91 & Fe XIII & $Y^{\text {later }}$ & 4 ; quiet phase, small brightenings \\
\hline 9 & - & 10 & 9.56 & Fe XIII & $\mathrm{N}$ & 1 ; point-like brightening \\
\hline 10 & -120 & 6 & 9.34 & Fe XIII & $Y^{\text {later }}$ & $>2$; point-like brigntening \\
\hline \multicolumn{7}{|l|}{$14 / 11 / 07$} \\
\hline 1 & - & 5 & 9.41 & $\mathrm{Fe} x \mathrm{v}$ & $Y^{\text {later }}$ & $>2$ large jets, pre-activation phase \\
\hline 2 & -2 & 2 & 9.09 & $\mathrm{Fe}$ XIII & $Y^{\text {later }}$ & $>1$, evolving loops of a faint $\mathrm{BP}$ \\
\hline 3 & -87 & 182 & 8.97 & $\mathrm{Fe}$ XIII & $Y^{\text {small }}$ & $>3 ;$ small BP \\
\hline 4 & -270 & 15 & 10.11 & Fe xxIII & $\mathrm{Y}$ & $>3,2$ observed jets \\
\hline 5 & - & 2 & 9.25 & Fe XvI & Ylater $^{\text {lat }}$ & $>3$, long-lived $\mathrm{BP}$ \\
\hline 6 & - & 2 & 9.14 & Fe xv & $\mathrm{Y}^{\text {later }}$ & $>2$, long-lived $\mathrm{BP}$ \\
\hline 8 & - & 10 & 9.23 & Fe XIII & $\mathrm{N}$ & $>2$; point-like brightening \\
\hline 9 & - & 13 & 9.21 & Fe XIII & $\mathrm{N}$ & $>2$; appears as brigtenings in a small-scale loop \\
\hline \multicolumn{7}{|r|}{ 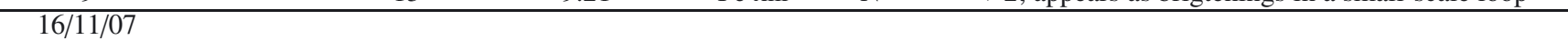 } \\
\hline 1 & -5 & 6 & 9.15 & $\mathrm{Fe} x \mathrm{v}$ & Ylater $^{\text {la }}$ & 2; $\mathrm{BP}$ in a quiet phase \\
\hline 2 & - & 8 & 9.07 & $\mathrm{Fe}$ XIII & $Y^{\text {later }}$ & $>1 ; \mathrm{BP}$ in a quiet phase \\
\hline 3 & -2 & 5 & 9.03 & Fe XIII & $\mathrm{N}$ & $>2$; quiet $\mathrm{BP}$ \\
\hline 4 & -3 & 3 & 8.89 & Fe XIII & $Y^{\text {later }}$ & 1; faint loop brightenings \\
\hline 5 & -2 & 2 & 8.55 & Fe XIII & $\mathrm{N}$ & $>1$; point-like $\mathrm{BP}$ \\
\hline 6 & - & 2 & 8.98 & Fe XIII & $\mathrm{N}$ & $>3$; small BP, quiet phase \\
\hline 7 & - & 12 & 9.03 & Fe XIII & $\mathrm{N}$ & 3; point-like brightening \\
\hline 8 & -11 & 6 & 8.84 & $\mathrm{Fe} x \mathrm{v}$ & $Y^{\text {later }}$ & 1 large, small AR \\
\hline 10 & -5 & 8 & 8.80 & $\mathrm{Fe} x \mathrm{v}$ & $\mathrm{Y}^{\text {small }}$ & $>3$; large $\mathrm{BP}$ in a quiet phase \\
\hline 11 & -3 & 4 & 8.82 & Fe XIII & $\mathrm{N}$ & $>2$; point-like $\mathrm{BP}$ \\
\hline
\end{tabular}

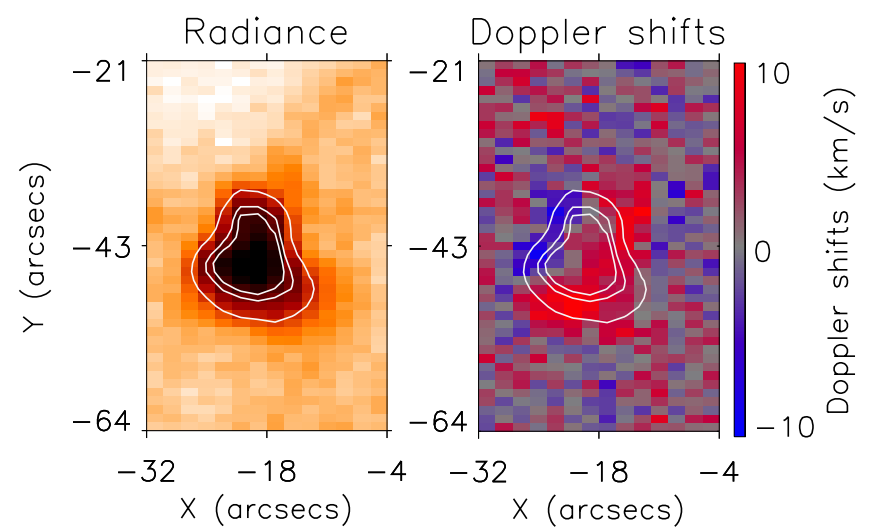

Fig. 6. Event No. 1 on January 13. The raster was taken in the Fe XII $195 \AA$ line between 15:01 UT and 15:12 UT.

a blue-shifted emission and the other half a red-shifted emission. These Doppler shifts are well known to indicate up-flows and down-flows of both sides of loops. However, as discussed in Young et al. (2012), blue/red-shift Doppler patterns can often occur due to strong intensity gradients. They are caused by an asymmetric point spread function (PSF) that is discussed in detail in Young et al. (2012). To avoid a mis-interpretation, we cross-checked each Doppler shift with imaging information from XRT. If the image sequence did not confirm a plasma proper motion while Doppler shift was measured, this Doppler shift was not considered as real but rather instrumental (PSF). The BP had several episodes of microflaring, which resulted in the BP becoming larger with small loops rising and then shrinking back to their original size. None of the microflaring resulted in the formation of an EUV/X-ray jet.

The event in Fig. 7 is a very clear example of a brightening followed by an expanding loop. This event, however, suffered by a gap of 9 min in the XRT observations. Nevertheless, we clearly see that a brightening in a region of a few pixels is followed by a loop expansion originating from this region. Similar examples will be studied best in the future using AIA (The Atmospheric Imaging Assembly) data where rising and shrinking of loops can be separated from cooling and heating thanks to the unprecedented cadence of the instrument as well as its coverage of numerous passbands. The blue- and red-shift patterns in Fig. 7 are 
Table 7. Physical properties of the brightening events observed by SUMER.

\begin{tabular}{|c|c|c|c|c|c|c|}
\hline $\begin{array}{l}\text { Date } \\
\text { Identity }\end{array}$ & Event No. & $\begin{array}{l}\text { Doppler shifts, } \\
\text { Bue }\end{array}$ & $\begin{array}{c}\mathrm{Ov}\left(\mathrm{km} \mathrm{s}^{-1}\right) \\
\text { Red }\end{array}$ & $\begin{array}{l}\mathrm{S}_{\mathrm{II}} / \mathrm{C}_{\mathrm{I}} / \mathrm{Si} \text { II } \\
\text { (response) }\end{array}$ & $\begin{array}{c}\text { Mg x } \\
\text { (response) }\end{array}$ & $\begin{array}{l}\text { Flow/outflow } \\
\text { Remarks }\end{array}$ \\
\hline \multicolumn{7}{|l|}{$12 / 11 / 07$} \\
\hline $\mathrm{CH}$ & S1 & $-136 /-70$ & 16 & Y & $\mathrm{N}$ & outflows, $>3$, SS \\
\hline $\mathrm{CHB}$ & S2 & -7 & $13 / 104$ & $\mathrm{Y}$ & $\mathrm{N}$ & outflows,SS; brightenings in small-scale loops \\
\hline $\mathrm{CHB}$ & S3 & $-139 /-65$ & 28 & $\mathrm{Y}$ & $\mathrm{N}$ & SS; BP \\
\hline $\mathrm{CH}$ & S4 & $-85 /-66 /-54$ & 23 & - & - & $\mathrm{R}$; small-scale brightening \\
\hline $\mathrm{CH}$ & S5 & $-150 /-80$ & $100 / 24$ & Y & Y & outflow, $>3$, SS, $(-90,-245)$, large active BP \\
\hline $\mathrm{CHB}$ & S6 & $-50 /-21$ & 12 & Y & $\mathrm{N}$ & $>1$, SS, $(-100,-320)$, point-like brightening \\
\hline $\mathrm{CH}$ & S7 & $-68 /-34$ & $26 / 17$ & - & - & $>2, \mathrm{R},(-130,-275)$; short-lived small-scale BP \\
\hline $\mathrm{CH}$ & 1 & - & $70 / 17$ & - & - & R; post jet downflow \\
\hline $\mathrm{CHB}$ & 7 & -150 & 50 (Si IV) & - & - & SS \\
\hline $\mathrm{CH}$ & 8 & -36 & 22 & - & - & $\mathrm{R}$ \\
\hline \multicolumn{7}{|l|}{$14 / 11 / 07$} \\
\hline $\mathrm{CH}$ & 3 & $-77 /-53$ & - & - & - & $\mathrm{R}$ \\
\hline $\mathrm{CHB}$ & 4 & -240 & 60 & $\mathrm{Y}$ & $\mathrm{Y}$ & SS \\
\hline- & $4 a$ & $-127 /-56$ & 7,80 & & & \\
\hline $\mathrm{CHB}$ & 5 & -85 & 36 & Y & $\mathrm{N}$ & SS \\
\hline $\mathrm{CHB}$ & 6 & $-23 /-85$ & 31 & Y & Y & SS \\
\hline $\mathrm{CHB}$ & 9 & -64 & 52 & - & - & $\mathrm{R}$ \\
\hline $\mathrm{CHB}$ & S1 & -99 & 58 & - & - & $\mathrm{R}$ \\
\hline \multicolumn{7}{|l|}{$16 / 11 / 07$} \\
\hline $\mathrm{CH}$ & 5 & $-60 /-25$ & - & - & - & $\mathrm{R}$ \\
\hline $\mathrm{CHB}$ & 10 & -58 & - & - & $\mathrm{R}$ & \\
\hline $\mathrm{CH}$ & 11 & -94 & 30 & - & - & $\mathrm{R}$ \\
\hline QS & 9 & $-20 /-7$ & & Y & $\mathrm{N}$ & SS \\
\hline AR & S1 & $-57 /-34$ & 19 & - & - & outflow, $>2, \mathrm{R},(-705,-140)$ \\
\hline
\end{tabular}

Notes. The Mg x spectral window, which also includes Si ii and C I, was transferred to the ground only when SUMER was in sit-and-stare (SS) mode. "R" refers to a rastering mode. The events that were not co-observed with EIS on November 12 and 14 are marked with "S" followed by a digit. The co-ordinates of the events not marked in Fig. 1 are given in the "Remarks" column with " $(X X, Y Y)$ " in unit of arcsec.

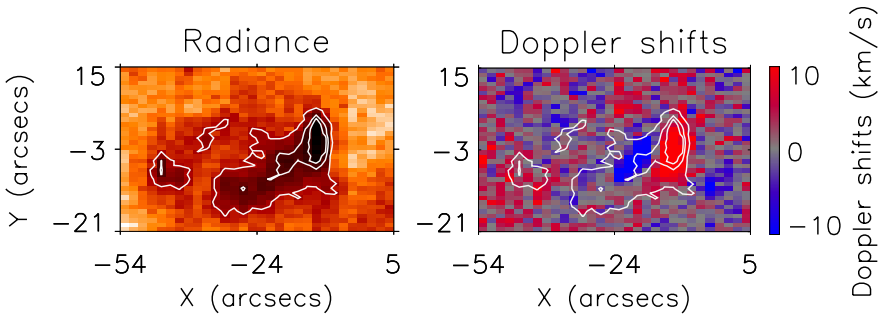

Fig. 7. Event No. 2 on January 13. The raster was taken in the Fe XII $195 \AA$ Aine between 14:35 UT and 14:55 UT.

difficult to interpret but they seem to be emitted from the rising and then contracting loop, as seen in the XRT image sequence. Note that an exposure time of $40 \mathrm{~s}$ was used here, which is relatively long with respect to the dynamics of the observed phenomena.

The plasma properties of the events found in the quiet Sun are listed in Table 5. The Doppler shifts are measured as an average over the blue- or red-shift pattern associated with the event. The electron density was calculated over only the bright pixels to obtain sufficient signal-to-noise. Each spectral line was investigated for a response in the studied event and thus the maximum temperature of the event was defined. The temperatures and densities of the quiet Sun events are discussed in the next section.

In general, the QS phenomena evolve dynamically, i.e. small-scale structural changes are seen at any time. Despite this "activity", however, none of the QS events has been identified as a jet-like event (see the definition in Sect. 1). The average Doppler shifts of the QS events are a blue-shift of $-3.6 \mathrm{~km} \mathrm{~s}^{-1}$ and a red-shift of $4.4 \mathrm{~km} \mathrm{~s}^{-1}$ derived from a single Gaussian fit.
One should bear in mind that these are not absolute values (see Sect. 2.1 for more details) but rather give an indication of the velocity range of QS brightening events. Of 15 brightenings, five show flows/outflows, i.e. plasma is seen to move away from the initial source brightening. In all cases, however, the ejected plasma remains trapped in loops. In one event the outflow was observed in the XRT images after the EIS rastering of this region was completed. The events that are not seen to produce a flow or outflow in the XRT images appear as a compact brightening that maybe caused by their intricate structure or simply insufficient instrumental resolution. Likely, that in some cases the flows/outflows had far too low emission and, therefore, could not be separated from the background emission. A future multichannel AIA study will be able to confirm this possibility. All events occurred several times, as visually estimated from their XRT lightcurves (for more details on this see Paper II). We do not give a precise number of the occurrence rate because that would require a definition of a time and intensity threshold, which is not a subject of this study and has been provided in detail in Paper II. This information, however gives an indication of the dynamics of the events studied here.

\subsubsection{Temperatures and electron densities}

The temperature of the quiet-Sun events is defined by the maximum formation temperature of the spectral lines in which these events were registered (Table 5). All observed spectral lines were investigated for a response in each event. Of 15 events, 4 are seen in Fe xv $\left(\log T_{\max } \approx 6.3 \mathrm{~K}\right)$ and 11 in Fe XIII $\left(\log T_{\max } \approx 6.2 \mathrm{~K}\right)$. None of the events showed a signature in spectral lines with formation temperatures higher than the formation temperature of 
M. S. Madjarska et al.: Coronal hole boundaries at small scales. III.

Fe xv. These temperatures refer in most cases to the energy deposition site (the pixels with the highest intensity), which always has the highest temperature.

The electron density was obtained from the spectral line ratio of Fe XII $186.88 \AA$ to $195.12 \AA$ (for more details see Madjarska 2011) and describes plasmas at temperatures $\log T_{\max } \approx 6.1 \mathrm{~K}$, which is the maximum formation temperature of Fe XII. The quiet-Sun events have an average electron density of $\log _{10} N_{\mathrm{e}} \approx$ $8.87 \mathrm{~cm}^{-3}$. In only four cases, the electron density is above $\log _{10} N_{\mathrm{e}} \approx 9.0 \mathrm{~cm}^{-3}$ (Table 5: event 7 on Nov. 9, event 1 on Jan. 10 and events 1 and 4 on Jan. 13). Event 7 is a BP clearly present in X-rays. The measurement of the electron density for event 1 on Jan. 10 were taken during the peak of the intensity as defined from the X-rays light-curve. The two events 1 and 4 on Jan. 13 are BPs.

\section{2. $\mathrm{CH}$ and $\mathrm{CHB}$ brightenings}

\subsubsection{Structure and dynamics}

The $\mathrm{CH}$ events, 35 in total, were concentrated at the coronal hole boundaries and inside coronal holes where pre-existing or newly emerging loop structures known as coronal bright points are found. Of 35 events 23 , i.e., $65 \%$ were seen to produce outflows that in most cases represent jet-like phenomena called EUV/X-ray jets, as observed in XRT images. Several features were found to be common for all observed events. All were associated with coronal bright points either pre-existing or newly emerging at X-ray temperatures. They evolve dynamically and produce numerous outflows (jet-like phenomena) until the full disappearance of the BP. Multiple energy deposition during a single event is a common feature. After each jet, the BP becomes smaller or even invisible at X-ray temperatures. In several cases BPs are not visible at XRT temperatures prior to the transient event because they are formed of cooler lower lying loops. We refer to these BPs as newly emerging. These BPs would appear as a small-scale (only a few pixels) brightening in the XRT images, and a few minutes or even seconds prior the occurrence of the X-ray jet. After the jet, the BP will again become invisible at X-ray temperatures. Several events of this type can be followed in the online material for the four days of coronal hole observations (see Figs. A.1 and A.2). All events follow the same evolutionary scenario, i.e., a sudden brightening (microflaring) in a BP is followed by plasma ejections as in the case described in Madjarska (2011).

To obtain the Doppler shifts in high-velocity events, we used a multi-Gaussian fit (double or triple) for EIS Fe XII $195.12 \AA$ and SUMER O v $629.77 \AA$. The largest red- and blue-shifts correspond to the peak of the Gaussian fit in the corresponding wing of the line. The wings extended to even higher velocity but the low signal does not permit a Gaussian fit. In most of the cases we were also able to fit the blend by Fe xII $195.18 \AA$ (Young et al. 2009). The average Doppler shifts of the $\mathrm{CH}$ events are a blue-shift of $-160 \mathrm{~km} \mathrm{~s}^{-1}$ and red-shift of $17 \mathrm{~km} \mathrm{~s}^{-1}$. These high Doppler velocities clearly indicate the more dynamic nature of $\mathrm{CH} / \mathrm{CHB}$ events with respect to the QS phenomena. In all cases, the high Doppler shifts were found only in the jet-like events. This strongly suggests that the co-existence of open and closed magnetic fields is a necessary condition for plasmas to be accelerated to high velocities.

Interestingly, the number of registered jets has decreased while the coronal hole was progressing through the visible solar disk towards the limb. One possible explanation is that the change of the line-of-sight is the cause. Another possibility is
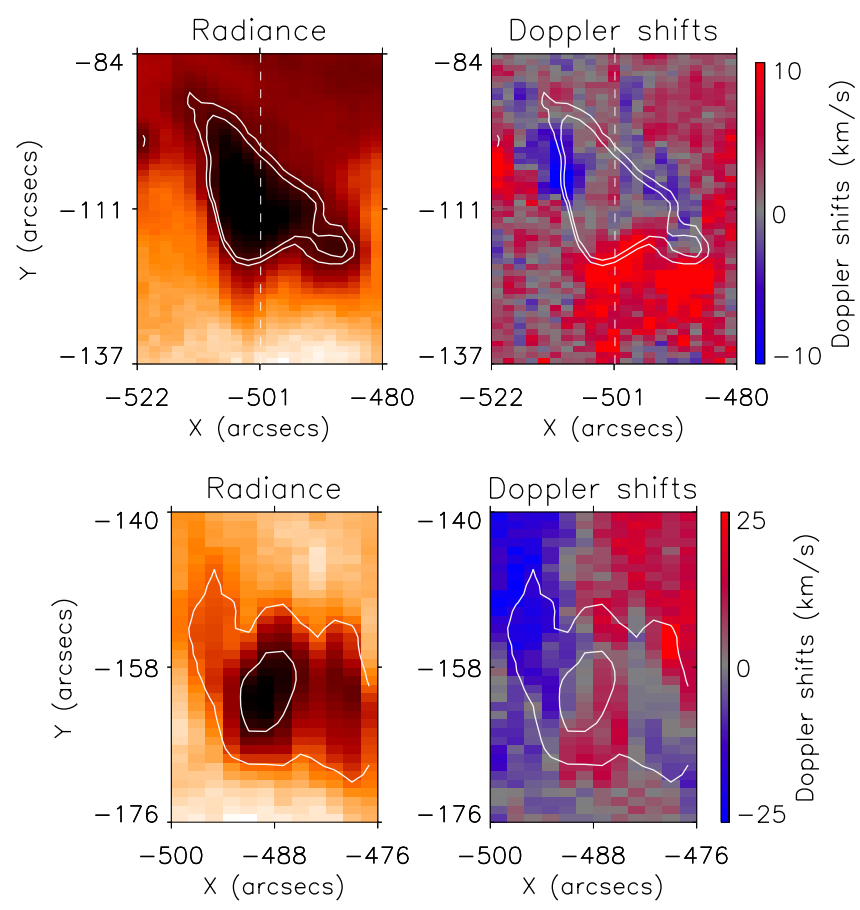

Fig. 8. Two examples, event 9 (top) and 11 (bottom) of brightening events observed on November 9. The left and right panels represent the Fe XII $195 \AA$ intensity and Doppler shift images, respectively. The event 9 raster is taken between 07:03 UT and 07:24 UT and the raster for event 11 is from 11:47 UT to 11:59 UT (see the online material to follow its full evolution). Event 9 is at the coronal hole boundary while event 11 is inside the coronal hole. White dashed lines indicate the SUMER slit sit-and-stare position. The radiance images are plotted in negative logarithmic scale.

a decrease of ephemeral region emergence in the coronal hole with time but this has never been reported or studied as far as we are aware of.

In Figs. 8-11 we give examples from each day of the coronal hole observations. The brightening event (No. 9) shown in Fig. 8 (top) happens in a long-living large bright point at the coronal hole boundary. It produced several outflows, two of which were significantly large. The BP structure was present during the entire observing period and it is fully in the EIS FOV only during the large raster. The dynamic evolution of the BP can be followed best in the SUMER sit-and-stare observations. The SUMER slit lies across the multi-loop structure of the BP as shown in Fig. 8. The evolution of the feature can be seen in the online material. The simultaneous coverage of this event by SUMER and EIS demonstrates once again the poor signal in the Mg x line while the EIS Fe XII shows a very strong increase (for more details on this problem see Madjarska 2011).

The brightening No. 11 (Fig. 3, first panel) shown in Fig. 8 (bottom) represents a typical BP and has the same size and shape as the QS BP shown in Fig. 6. It shows a well-pronounced halfblue and half-red Doppler shift pattern. In the quiet phase this pattern is probably mostly due to a PSF effect, although flows from one footpoint to the other are certainly present. The physical and instrumental effects are hard to separate. This BP shows higher density, temperature and far larger Doppler shifts with respect to the QS BP shown in Fig. 6. The QS BP did not produce strong outflows during the time it was scanned by EIS. There is a small probability that it could have had higher values for the plasma parameters if the measurements had been taken during higher activity. However, we have to note that even when EIS 

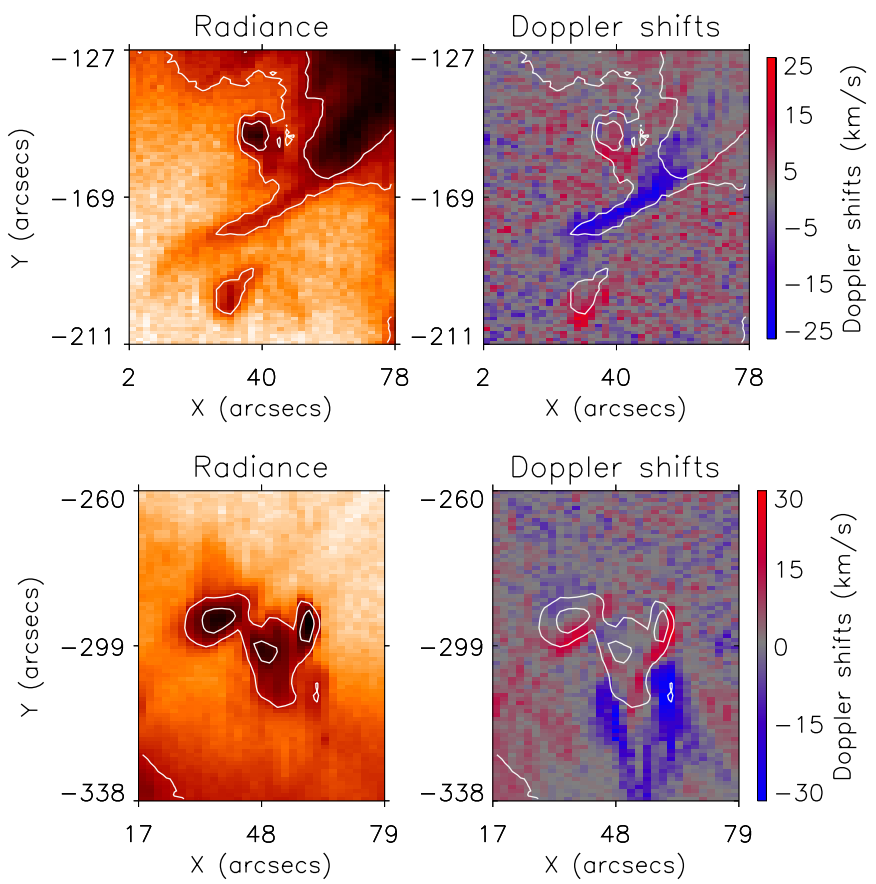

Fig. 9. Two examples, brightening event 3 at coronal hole boundaties (top) and 1 in coronal hole (bottom), observed on November 12. The left and right panels represent the Fe XII $195 \AA$ intensity and Doppler shift images, respectively. Event 3 was scanned between 01:17 UT and 01:54 UT while event 1 between 01:17 UT and 01:49 UT during the big raster observations. The top event happened at the coronal hole boundary while the bottom inside the coronal hole.

was rastering events in the QS with strong flows, none of these events has reached the values of the plasma parameters of the jet-producing events in $\mathrm{CH} / \mathrm{CHBs}$.

In Fig. 9 we present examples of brightening caused by typical X-ray jets from coronal BPs at the boundary of a coronal hole (event No. 3) and inside a coronal hole (event No. 1). Event No. 3 produced one very faint but high velocity jet. After the jet the brightening (BP) in its footpoints disappeared at X-ray temperature and no more jets happened during the entire observing period. Event No. 1 started before the beginning of our observations, therefore we missed the time of the energy deposition but we were able to follow the X-ray jet phase in full detail. Two main flows are present, the outflow forming the jet and downflow in the footpoints associated with the BP. The pattern is identical to the example shown in Madjarska (2011). The BP produced two more jets becoming smaller after each jet, until it fully disappeared.

From November 14 we present two events that happened in a quiet phase during the EIS scan (Fig. 11). Their Doppler shifts are very low at coronal temperatures though the transition region $\mathrm{O} v$ line shows significant dynamics (see Sect. 4 for more details). The November 16 events (Nos. 8 and 1) are in a quiet phase during the EIS scans but later they produce large outflows that were unfortunately not detected by EIS.

\subsubsection{Temperatures and electron densities}

Only one event from the $\mathrm{CH} / \mathrm{CHB}$ events was registered in Fe xxIII (12 MK, event 4 on Nov. 14) measured in the flaring site (for more details see Madjarska 2011). This event produced a large jet with a temperature of maximum 2.5 MK. High temperatures of $2.5 \mathrm{MK}$ (Fe xvI; 1 event) and $2 \mathrm{MK}$ (Fe xv; 11 events) were found mostly in long-living BPs or the footpoints of large
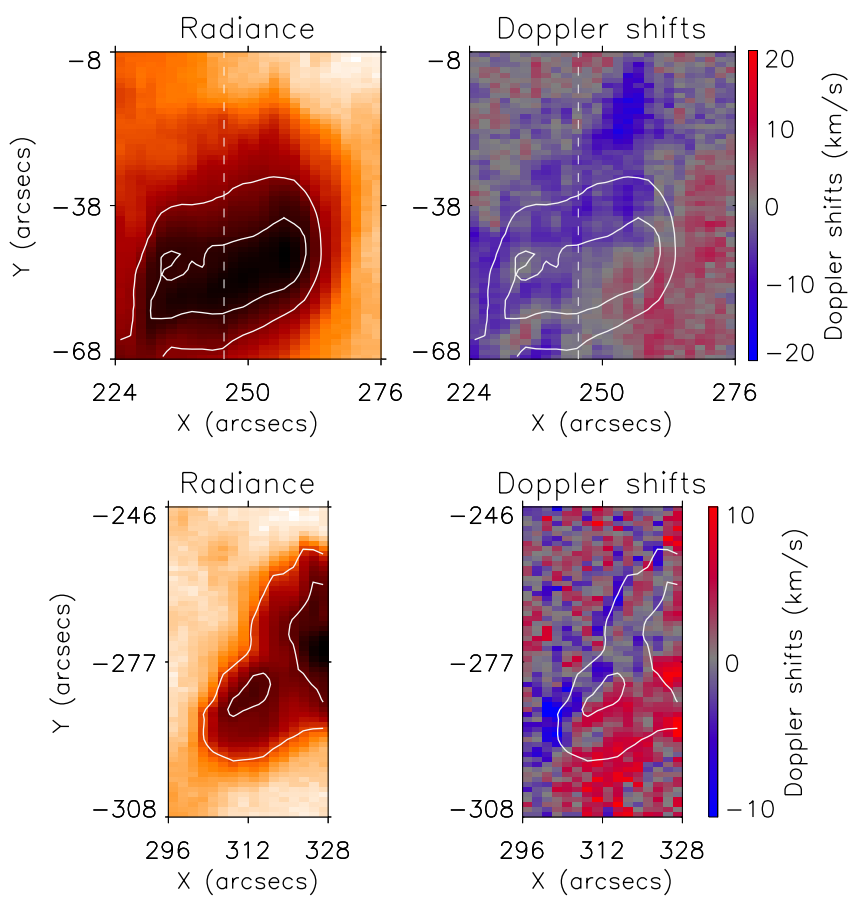

Fig. 10. Two examples of brightening events observed on November 14 (top: event 6 at a coronal hole boundary; bottom: event 1 inside the coronal hole). The left and right panels represent the Fe XII $195 \AA$ Antensity and Doppler shift images, respectively. Event 6 was observed between 00:48 UT and 01:15 UT while event 1 from 00:17 UT until 00:40 UT.
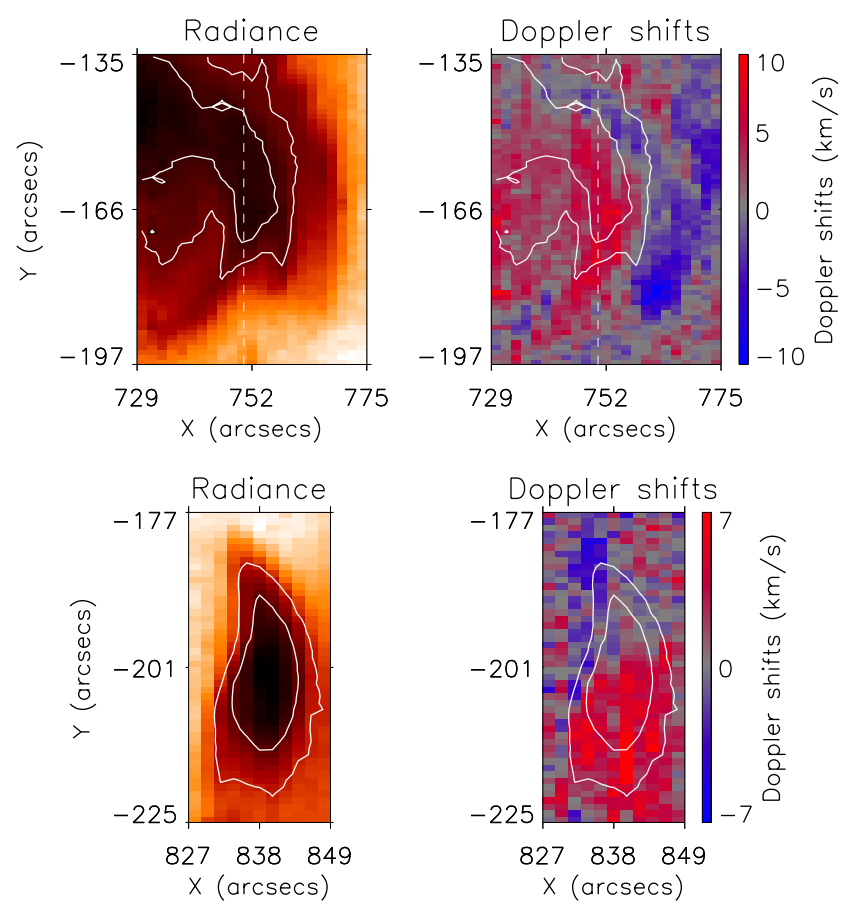

Fig. 11. Two examples of brightening events observed on November 16 (top: event 8 at a coronal hole boundary; bottom: event 1 inside the coronal hole). The left and right panels represent the Fe XII $195 \AA$ A intensity and Doppler shift images, respectively. Event 8 was scanned from 18:44 UT to 19:08 UT and event 1 from 18:08 UT to 18:18 UT.

jets (e.g. event 7 and 9 Nov. 12, event 4 Nov. 14). About $62 \%$ (22) of the events reached only the Fe XIII (1.6 MK) temperature. They are associated with jets, point-like brightenings, quiet 
phase of faint BPs (in X-rays), or brightenings in pre-existing loops.

The average electron density of all $\mathrm{CH} / \mathrm{CHB}$ events is $\log _{10} N_{\mathrm{e}} \approx 9.20 \mathrm{~cm}^{-3}$. The events that show electron densities above the average value are all associated with jet-producing phenomena and their densities correspond to the energy deposition site, not to the jets themselves. The average density of the energy deposition sites is $\log _{10} N_{\mathrm{e}} \approx 9.51 \mathrm{~cm}^{-3}$. Only in five cases were the electron densities measured in the jet itself (event 12 on Nov. 9, events 3, 6 and 7 on Nov. 12, event 3 on Nov. 14) and their average electron density is $\log _{10} N_{\mathrm{e}} \approx 8.76 \mathrm{~cm}^{-3}$.

\section{SUMER observations of the quiet Sun and $\mathrm{CH} / \mathrm{CHB}$ brightenings}

The present study provides an unprecedented analysis of EIS and SUMER co-observations combined with co-temporal imaging from XRT. Because the EIS spectrometer has very limited coverage of spectral lines with transition region formation temperatures, the simultaneous observations with SUMER contribute enormously to the understanding of the impact of these transient phenomena to the dynamics and temperature gradients in the solar atmosphere. In Table 7 we present the physical parameters of the brightenings that occurred under the SUMER slit. The SUMER observations from November 9 suffered instrumental problems (see Sect. 2.2) and are unsuitable for the present analysis. During part of the observations taken on November 12, the SUMER slit was pointed at a Solar_x coordinate, which was $96^{\prime \prime}$ east from the requested pointing. Nevertheless, we analysed these data, but they do not have EIS coverage.

All brightening events registered in SUMER were found to have a counterpart at chromospheric temperatures. We point out that in all cases the slit was crossing the footpoints of the transient brightenings, but not the jets. In a single case, event 7 on Nov. 12, the slit crossed the jet while SUMER was rastering in the Si Iv $1394 \AA$ line. The chromospheric response was associated with downflows as determined from the transition region $\mathrm{O}$ v $629 \AA$ and N v $1238 \AA$ lines. During the SUMER Mg x $625 \AA$ and EIS Fe xII $195 \AA$ co-observations, only a third of the events had a response in the $\mathrm{Mg}$ x $625 \AA$ line while all events had a signature in the Fe XII $195 \AA$ line. A discussion of the $\mathrm{Mg}$ x response can be found in Madjarska (2011). A significant part of the transition-region dynamics seen as Doppler shifts in the $\mathrm{O} v$ line has no counterpart at X-ray temperatures. We believe that spicules may be mostly responsible for this dynamics (Dere et al. 1983; Madjarska \& Doyle 2003). We intentionally did not include the analysis of the observations taken in the Si IV line. This line is formed at temperatures of $80000 \mathrm{~K}$ and would detect the upper-chromosphere/low-transition-region spicule activity. Therefore, it is difficult and often impossible to separate a line profile produced by events originating in the chromosphere from events that originated in the corona and leave their imprint in the transition region. However, we included the Si Iv line in the animation sequence for the purpose of continuity of the presentation. From the comparison of the Doppler-shift measurements shown in Table 7 with the measurements in the Fe XII line given in Table 6, we can clearly see that transient brightenings triggered by X-ray jets or just flows in loops contribute significantly to the dynamics of the transition region. Flows are seen at all temperatures from the chromosphere to the corona, which demonstrates the impact on the mass flow in the solar atmosphere of events associated with transient brightenings. We give

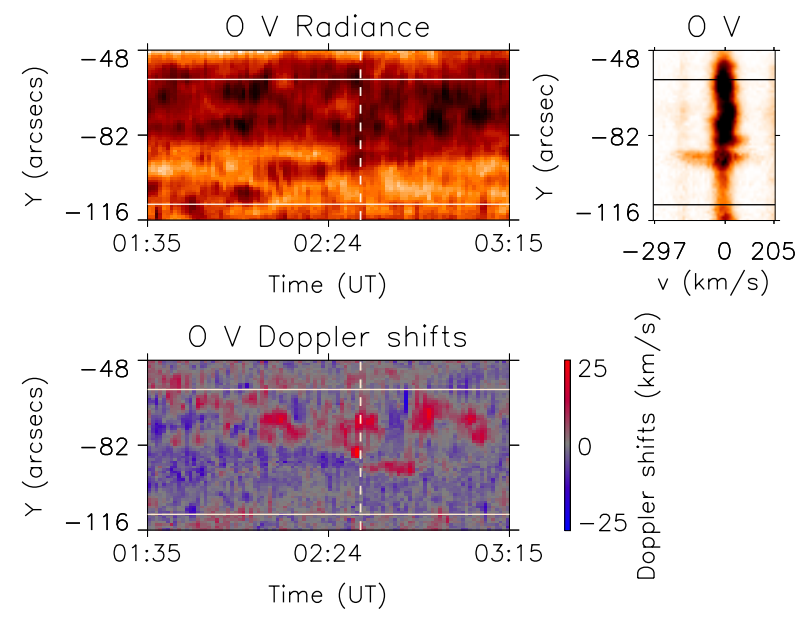

Fig. 12. SUMER sit-and-stare observations of the brightening event $\mathrm{S} 2$ (CHB) on November 12. The radiance (top) and Doppler (bottom) maps of the event are obtained in the O v $629 \AA$. The solid lines indicate the area of the event registered in X-rays. The last column from the top row shows the intensity along the slit at the time (02:34:35 UT) indicated with a dashed vertical line on the intensity image.

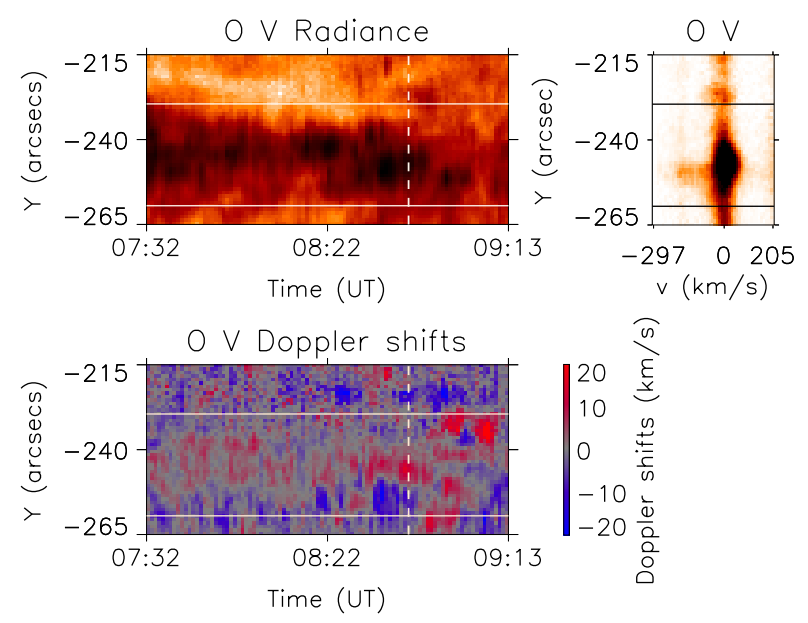

Fig. 13. Same as Fig. 12 for event S5 $(\mathrm{CH})$ on November 12. The time of the sample spectrum is 08:35:20 UT.

a few typical examples of events seen in the SUMER O v line and discuss their identification with well known phenomena from past spectrometer observations (HRTS and SUMER).

Typical examples of the SUMER O v line profiles produced by the transient brightenings associated with jets or flows in loops can be seen in Figs. 12 to 16 . The event S2, which is only seen in SUMER and XRT, evolved as a point-like brightening that produced faint X-ray jet(s) (the jet appearance is so faint in X-rays that it is difficult to judge how many jets have been produced). In response to this phenomenon, the $\mathrm{O} v$ line showed strong blue- and red-shifted emission typical for explosive events (Fig. 12). The Doppler shift appeared in a burst of a slightly more than $20 \mathrm{~min}$, which is often associated with EEs.

After the slit moved to a position that was $96^{\prime \prime}$ from the originally planned one, it stared at the edge of a large dynamically evolving BP. In Fig. 13 we show the intensity and Doppler-shift images of this phenomenon. Again, dynamically evolving emission in the wings of the $\mathrm{O} v$ line appears in all shapes associated with jets, flows, or explosive events (see the online material Fig. A.2). 

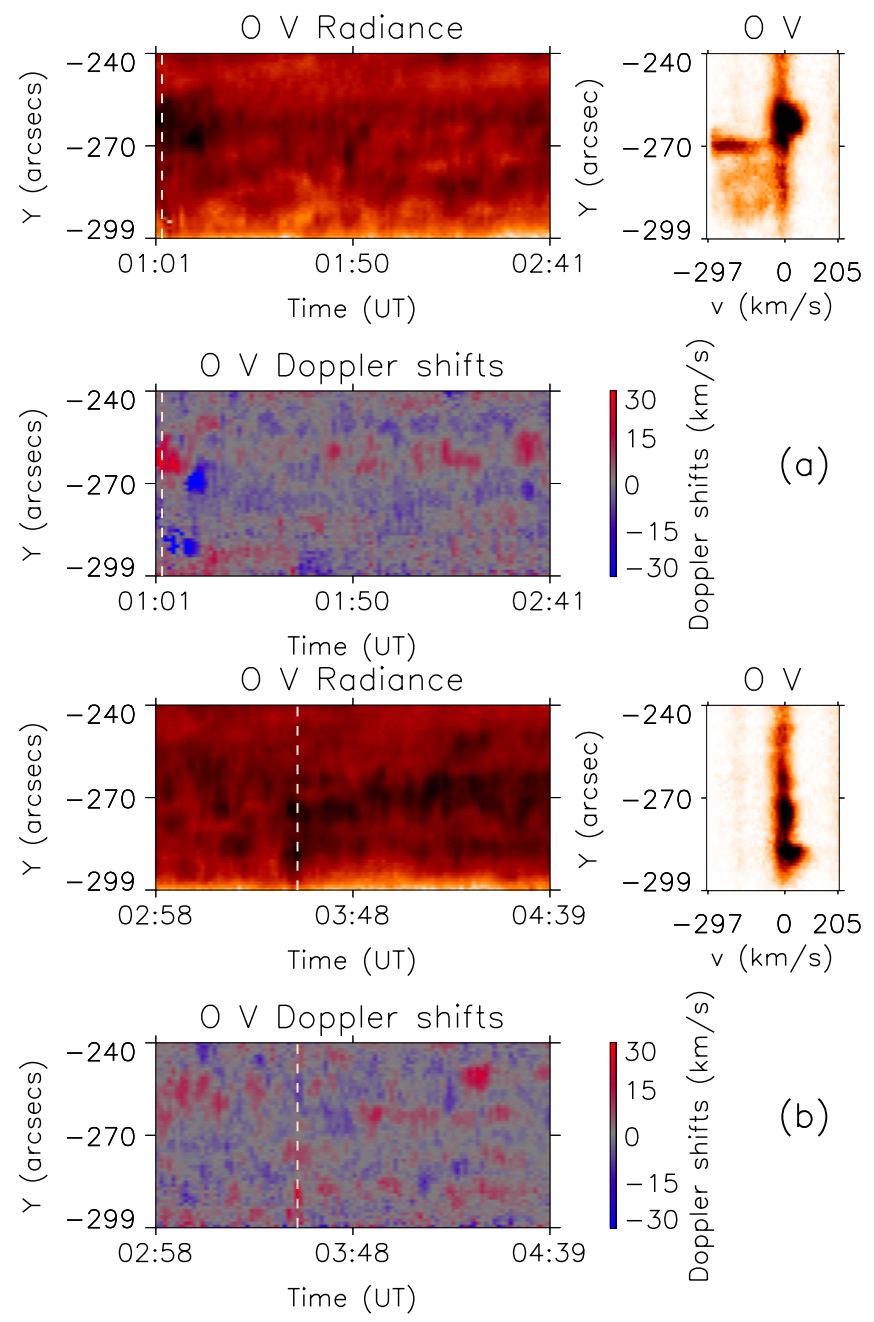

Fig. 14. Same as Fig. 12 showing event 4 (CHB) on November 14. The times of the sample spectra are 01:02:19 UT and 03:34:32 UT.

The next example given in Fig. 14 is the event studied in detail by Madjarska (2011). We show here the reoccurrence of the jet in the same BP (mentioned but not discussed in Madjarska 2011). During the first jet, the slit was positioned on the propagation path of the jet, while during the second jet, the slit crossed the opposite edge of the BP. The first jet (Fig. 14a) is seen as a very strong blue-shifted emission and the downflow as a redshifted emission in the $\mathrm{O} v$ line. At the time of the second jet, only the downflow can be seen (Fig. 14b) because the SUMER slit does not stare at the jet (see the online material for more details).

The example given in Fig. 15 occurred at the coronal hole boundary. The event is hardly seen to evolve during the EIS big image scan. In the SUMER sit-and-stare observations, however, the dynamics is clearly visible as varying blue- and red-shifted emission (see the November 14 animation for more details).

The last example given in Fig. 16 is the only quiet-Sun event recorded in the SUMER data. Brightenings along the SUMER slit with blue and red-shifted emission that do not exceed $50 \mathrm{~km} \mathrm{~s}^{-1}$ are seen during the event (see the November 16 animation for more details).

\section{Discussion and conclusions}

Knowing the plasma properties of small-scale transient phenomena is crucial for their modelling as well as the understanding
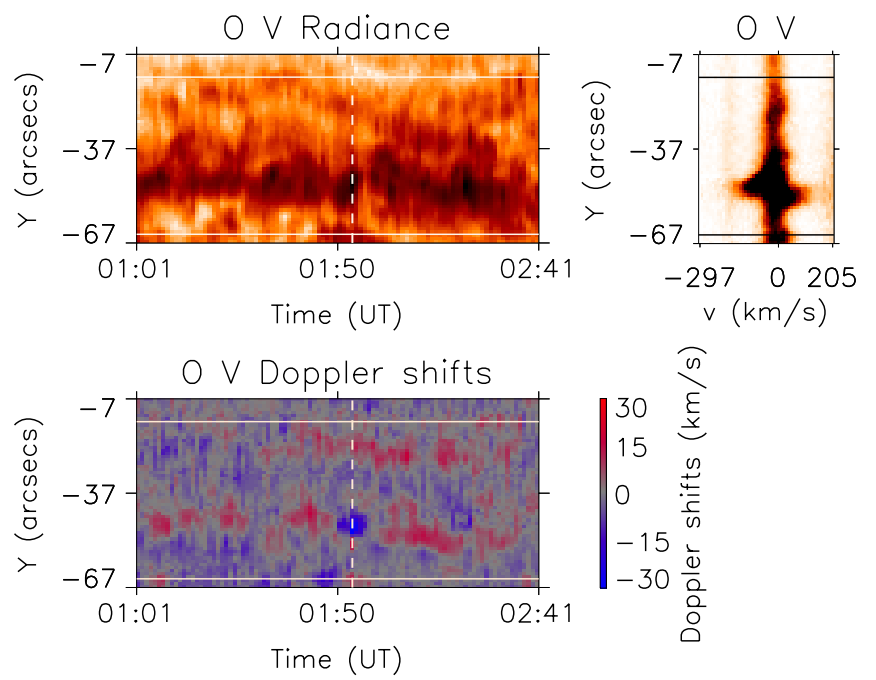

Fig. 15. Same as Fig. 12 showing event 6 (CHB) on November 14. The time of the sample spectrum is 01:55:13 UT.
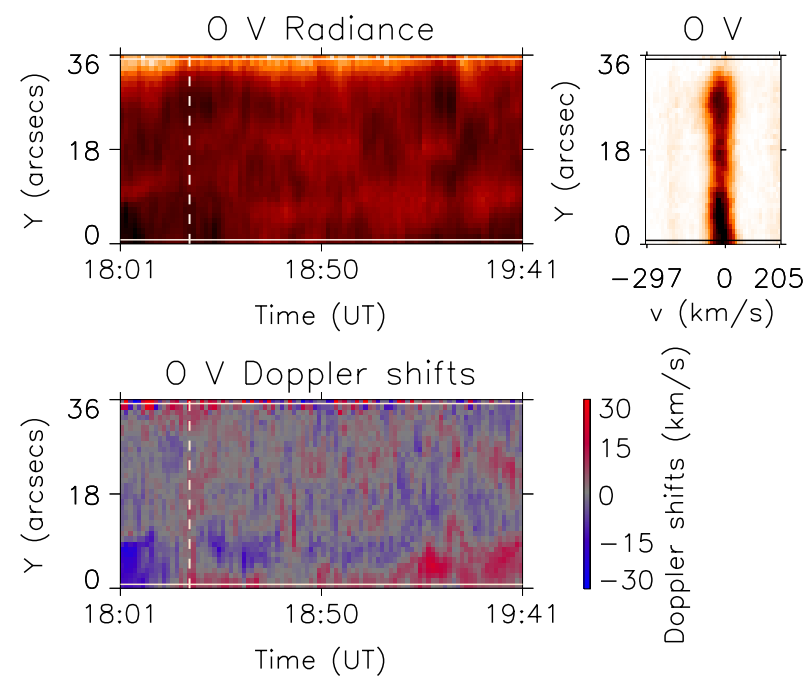

Fig. 16. Same as Fig. 12 showing event 9 (QS) on November 16. The time of the sample spectrum is 18:18:09 UT.

of their role in coronal heating and solar wind generation. The present paper delivered a follow-up spectroscopic study on many of transient small-scale events in coronal holes and their boundaries. The counterparts of these phenomena in the quiet Sun were also analysed.

During the past decade several groups (Shimojo \& Shibata 2000; Culhane et al. 2007b; Savcheva et al. 2007; Doschek et al. 2010) studied the properties of X-ray jets. First, Shimojo $\&$ Shibata (2000) investigated the physical parameters of coronal jets following their discovery in images from SXT/Yohkoh. Doschek et al. (2010) obtained electron density of $\log _{10} N_{\mathrm{e}} \approx$ $8.85 \mathrm{~cm}^{-3}$ at the base of a jet using the same line ratio as in the present study. The electron density of jets $\log _{10} N_{\mathrm{e}} \approx 8.76 \mathrm{~cm}^{-3}$ measured here is very close to their value. Furthermore, the present study provides measurements of the electron density in the flaring site, which is on average $\log _{10} N_{\mathrm{e}} \approx 9.51 \mathrm{~cm}^{-3}$. We derived similar differences in the electron density measured in the microflaring and flow/outflow sites in quiet-Sun transients, which strongly suggests that the same physical mechanism may trigger small-scale transients anywhere in the solar atmosphere. Shimojo \& Shibata (2000) obtained the electron densities of 
16 jets using the soft $\mathrm{X}$-ray imager onboard Yohkoh. They measured electron densities of $\log _{10} N_{\mathrm{e}} \approx 9.73 \mathrm{~cm}^{-3}$ in the microflaring sites and $\log _{10} N_{\mathrm{e}} \approx 9.23 \mathrm{~cm}^{-3}$ for the jets. These values are $60 \%$ higher for the energy deposition site and almost twice as high as for the jets compared to the values obtained here. The differences probably come from the filling factor assumptions with the imager data. We should also bear in mind that spectroscopic measurements of electron densities using spectral lines from the same element and ionization stage is the most reliable method for density diagnostics.

The present study used the widest temperature range coverage of small-scale transients thanks to the combination of SUMER and EIS observations, e.g. from $10000 \mathrm{~K}$ to $12 \mathrm{MK}$. Culhane et al. (2007b) found that the temperatures of BPs and jets are not higher than 2.5 MK (EIS slot observations) while Doschek et al. (2010) (EIS slit observations) obtained temperatures from 0.3 to $2.2 \mathrm{MK}$. Shimojo \& Shibata (2000) found similar temperatures of 3-6 MK for both the jets and flaring sites. Here, the jet temperatures reached a maximum of $2.5 \mathrm{MK}$ but in the majority of the cases the temperatures did not exceed 1.6 MK (Fe XIII). The footpoints of jets, which present large and long-lived coronal bright points seen in X-ray images, had maximum temperatures of $2.5 \mathrm{MK}$. The temperature of the flaring site of one event reached $12 \mathrm{MK}$. Only during this jet did the slit cross the flaring site a minute after the energy deposition took place. We believe that the higher temperature in the footpoints of jets must be a common property, but to confirm this, additional observational evidence is needed. No obvious difference was found between the temperatures of the quiet Sun and coronal hole transient events, pointing again towards the same physical mechanism for the origin.

The spectroscopic analysis of small-scale transient brightenings indicates that the majority of the phenomena that reach high coronal temperatures were triggered by multiple microflarings (during a single event), which take place in either the transition region or the corona, but no indication was found for energy deposition in the chromosphere. Chromospheric emission was mostly related to downflows in the footpoints of jets.

The similar temperatures and densities of the quiet Sun and coronal hole events, as well as the same observational signature of the onset of these events, i.e. microflaring, agree with the suggestion by Yokoyama \& Shibata (1995) that the physical origin of both jets ( $\mathrm{CH}$ events) and loop brightenings (typical for the QS) are possibly the same, i.e. magnetic reconnection. The only apparent difference is the higher dynamics of the $\mathrm{CH} / \mathrm{CHBs}$ events in terms of high-velocity plasma flows. This can be explained via the different magnetic field configuration involved. A reconnection of open and closed magnetic field lines in the $\mathrm{CHs}$ and at $\mathrm{CHBs}$ will result in the acceleration of the ejected plasma to higher speeds due to the lower density along the open field lines.

We can conclude that the dynamic day-by-day and even hour-by-hour small-scale evolution of coronal hole boundaries reported in Paper I is indeed related to coronal bright points. The XRT observations reported in Paper II revealed that these changes result from the dynamic evolution of coronal bright points that produce multiple jets during their lifetime until their full disappearance. Madjarska et al. (2004) first detected the spectroscopic signature of these dynamic changes. This signature, in the form of non-Gaussian profiles of spectral lines at transition region temperatures, was believed to be produced by the so-called "explosive events" (see Sect. 1 for details) with the strong blue- and red-shifted emission resulting from bidirectional jets expelled from a current sheet due to magnetic reconnection. The present study added the last pieces to the puzzle by revealing which phenomena do produce these bidirectional jets, their origin, evolution, temperatures, etc. We found that numerous jets from coronal bright points in coronal holes and at their boundaries do carry high-density plasma at very high speeds throughout the solar atmosphere. It is now known that many of these jets reach the interplanetary space (Paraschiv et al. 2010). More recently, Jackson et al. (during the SDO-4/IRIS/Hinode workshop 2012) reported their finding of "a positive correlation between the brightest of the polar jets and a high-speed response traced into the interplanetary medium".

The concentration of jet-like events in coronal holes and at their boundaries as shown in Paper II and their plasma characteristics obtained here make them an important candidate for one of the sources of the slow solar wind. Most recently, though, Neugebauer (2012) "proposed that the interplanetary manifestations of X-ray jets observed in solar polar coronal holes during periods of low solar activity are the peaks of the so-called microstreams observed in the fast polar solar wind". We already know that there is no physical difference between equatorial and polar jets, therefore, the possibility that X-ray jets propagate into the fast solar wind as microstreams should not be excluded.

The present study demonstrates the association of X-ray jets and flows in loops as one of the main phenomena that produce "explosive events". Teriaca et al. (2002) suggested that explosive events or rather events with strong non-Gaussian profiles do not reach coronal temperatures based on their response in the SUMER Mg x line. Note that the study was based on a few events only. The present study clearly shows that this line is not reliable for these diagnostics (see detailed discussion of this in Madjarska 2011). Therefore, the possibility that the events discussed here may significantly contribute to the temperature gradient in the solar atmosphere should be reconsidered. This subject needs to be revisited in the light of the results presented here, and new statistical studies based on imaging data (e.g. AIA on the Solar Dynamic Observatory) supported by spectroscopic information should re-evaluate the power distribution of these events. Recently, a multi-instrument study by Subramanian et al. (2012) of another phenomenon known as blinkers or transition region brightenings, which were known only by their appearance in spectroscopic data (mainly from the Coronal Diagnostics Spectrometer onboard SoHO) showed that they cover a wide range of phenomena. Blinkers were found to be the EUV response of various transient events originating at coronal, transition region, and chromospheric heights as are the explosive events. Hence, both blinkers and explosive events will contribute to the formation and maintenance of the temperature gradient in the transition region and the corona.

This study demonstrates the immense capabilities of the present imaging and spectroscopic instrumentation. It also shows how in the future the forthcoming IRIS (Interface Region Imaging Spectrograph) instrument can be successfully combined with the already existing imaging and spectroscopy instruments such as EIS/SOT/XRT/Hinode and AIA/HMI/SDO. The next part of this extensive study will present the magnetic field evolution (SOT/Hinode) associated with the small-scale transients discussed here.

Acknowledgements. We gratefully acknowledge the anonymous referee for the important correction and suggestions. Research at Armagh Observatory is grantaided by the N. Ireland Department of Culture, Arts and Leisure. We also thank STFC for support via grant ST/J001082/1. Hinode is a Japanese mission developed and launched by ISAS/JAXA, with NAOJ as domestic partner and NASA and STFC (UK) as international partners. It is operated by these agencies in 
co-operation with ESA and NSC (Norway). The research leading to these results has received funding from the European Commissions Seventh Framework Programme under the grant agreement No. 284461 (eHEROES project). The authors MM and JGD thank ISSI, Bern for the support of the team "Small-scale transient phenomena and their contribution to coronal heating".

\section{References}

Alexander, D., \& Fletcher, L. 1999, Sol. Phys., 190, 167 Brueckner, G. E., \& Bartoe, J.-D. F. 1983, ApJ, 272, 329

Culhane, J. L., Harra, L. K., James, A. M., et al. 2007a, Sol. Phys., 243, 19 Culhane, L., Harra, L. K., Baker, D., et al. 2007b, PASJ, 59, 751

Dere, K. P. 1994, Adv. Space Res., 14, 13

Dere, K. P., Bartoe, J.-D. F., \& Brueckner, G. E. 1983, ApJ, 267, L65

Dere, K. P., Bartoe, J.-D. F., \& Brueckner, G. E. 1989, Sol. Phys., 123, 41

Doschek, G. A., Landi, E., Warren, H. P., \& Harra, L. K. 2010, ApJ, 710, 1806

Doyle, J. G., Popescu, M. D., \& Taroyan, Y. 2006, A\&A, 446, 327

Golub, L., Deluca, E., Austin, G., et al. 2007, Sol. Phys., 243, 63

Innes, D. E., Inhester, B., Axford, W. I., \& Wilhelm, K. 1997, Nature, 386, 811

Ko, Y.-K., Raymond, J. C., Gibson, S. E., et al. 2005, ApJ, 623, 519

Krieger, A. S., Timothy, A. F., \& Roelof, E. C. 1973, Sol. Phys., 29, 505

Lemaire, P., Wilhelm, K., Curdt, W., et al. 1997, Sol. Phys., 170, 105

Madjarska, M. S. 2011, A\&A, 526, A19

Madjarska, M. S., \& Doyle, J. G. 2002, A\&A, 382, 319

Madjarska, M. S., \& Doyle, J. G. 2003, A\&A, 403, 731
Madjarska, M. S., \& Wiegelmann, T. 2009, A\&A, 503, 991

Madjarska, M. S., Doyle, J. G., \& van Driel-Gesztelyi, L. 2004, ApJ, 603, L57

Madjarska, M. S., Doyle, J. G., \& de Pontieu, B. 2009, ApJ, 701, 253

Moreno-Insertis, F., Galsgaard, K., \& Ugarte-Urra, I. 2008, ApJ, 673, L211

Neugebauer, M. 2012, ApJ, 750, 50

Paraschiv, A. R., Lacatus, D. A., Badescu, T., et al. 2010, Sol. Phys., 264, 365

Pariat, E., Antiochos, S. K., \& DeVore, C. R. 2010, ApJ, 714, 1762

Porter, J. G., \& Dere, K. P. 1991, ApJ, 370, 775

Savcheva, A., Cirtain, J., Deluca, E. E., et al. 2007, PASJ, 59, 771

Shibata, K., Ishido, Y., Acton, L. W., et al. 1992, PASJ, 44, L173

Shibata, K., Nitta, N., Strong, K. T., et al. 1994, ApJ, 431, L51

Shimojo, M., \& Shibata, K. 2000, ApJ, 542, 1100

Shimojo, M., Hashimoto, S., Shibata, K., et al. 1996, PASJ, 48, 123

Subramanian, S., Madjarska, M. S., \& Doyle, J. G. 2010, A\&A, 516, A50

Subramanian, S., Madjarska, M. S., Doyle, J. G., \& Bewsher, D. 2012, A\&A, 538, A50

Teriaca, L., Madjarska, M. S., \& Doyle, J. G. 2002, A\&A, 392, 309

Teriaca, L., Banerjee, D., Falchi, A., Doyle, J. G., \& Madjarska, M. S. 2004, A\&A, 427, 1065

Timothy, A. F., Krieger, A. S., \& Vaiana, G. S. 1975, Sol. Phys., 42, 135

Wang, Y.-M., Sheeley, Jr., N. R., Socker, D. G., et al. 1998a, ApJ, 508, 899

Wang, Y.-M., Sheeley, Jr., N. R., Walters, J. H., et al. 1998b, ApJ, 498, L165

Wilhelm, K., Curdt, W., Marsch, E., et al. 1995, Sol. Phys., 162, 189

Woo, R., Habbal, S. R., \& Feldman, U. 2004, ApJ, 612, 1171

Yokoyama, T., \& Shibata, K. 1995, Nature, 375, 42

Young, P. R., Watanabe, T., Hara, H., \& Mariska, J. T. 2009, A\&A, 495, 587

Young, P. R., O’Dwyer, B., \& Mason, H. E. 2012, ApJ, 744, 14 
M. S. Madjarska et al.: Coronal hole boundaries at small scales. III.
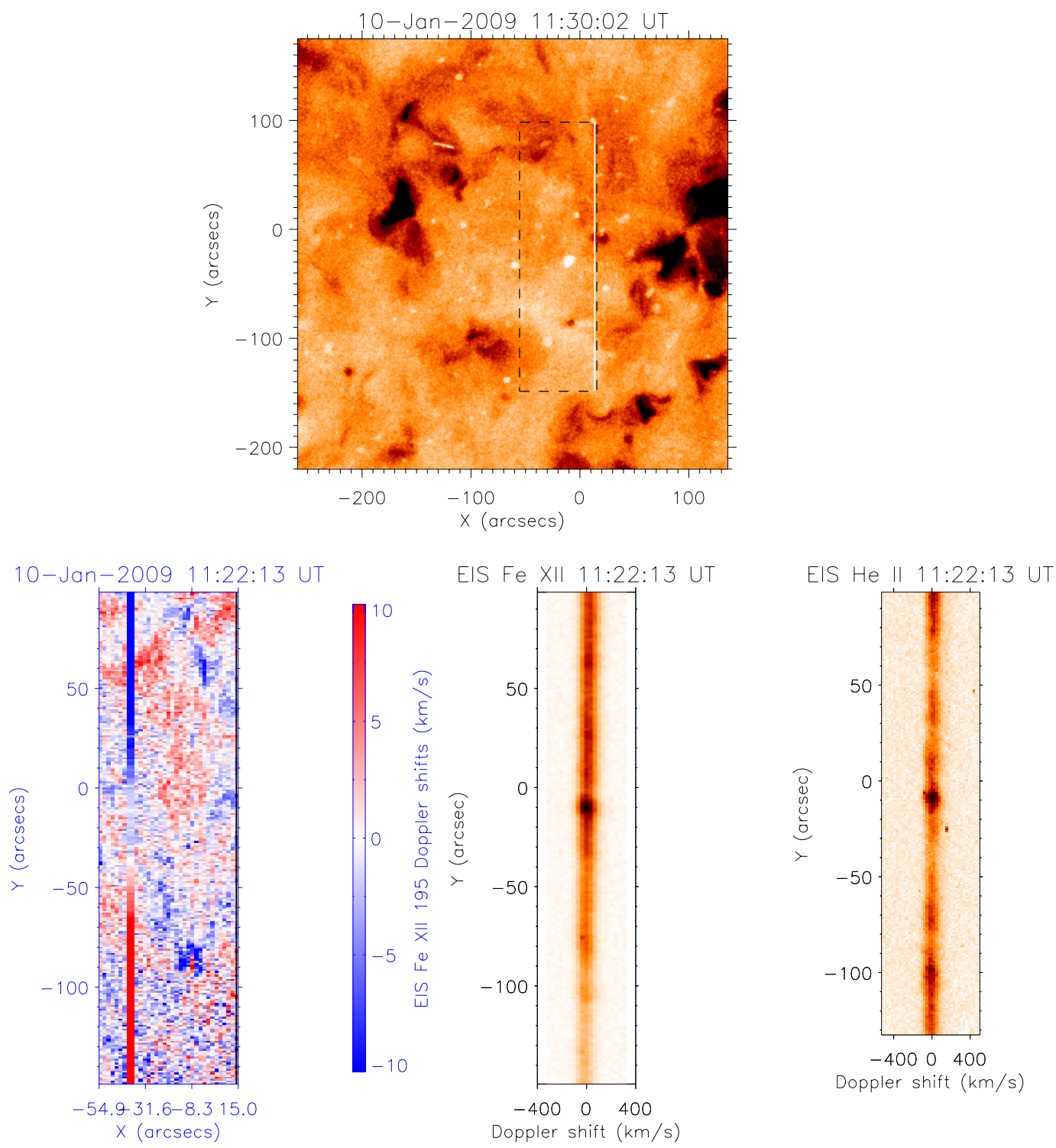

Fig. A.1. Animation of co-temporal images of the quiet-Sun dataset on January 10 including XRT images at the top with the EIS raster FOV overplotted with dashed lines and the EIS slit with solid white line at the given time. Bottom from left to right: EIS Doppler maps, Fe xII $195.12 \AA$, and He II 256.32 Å intensity along the slit. 
A\&A 545, A67 (2012)
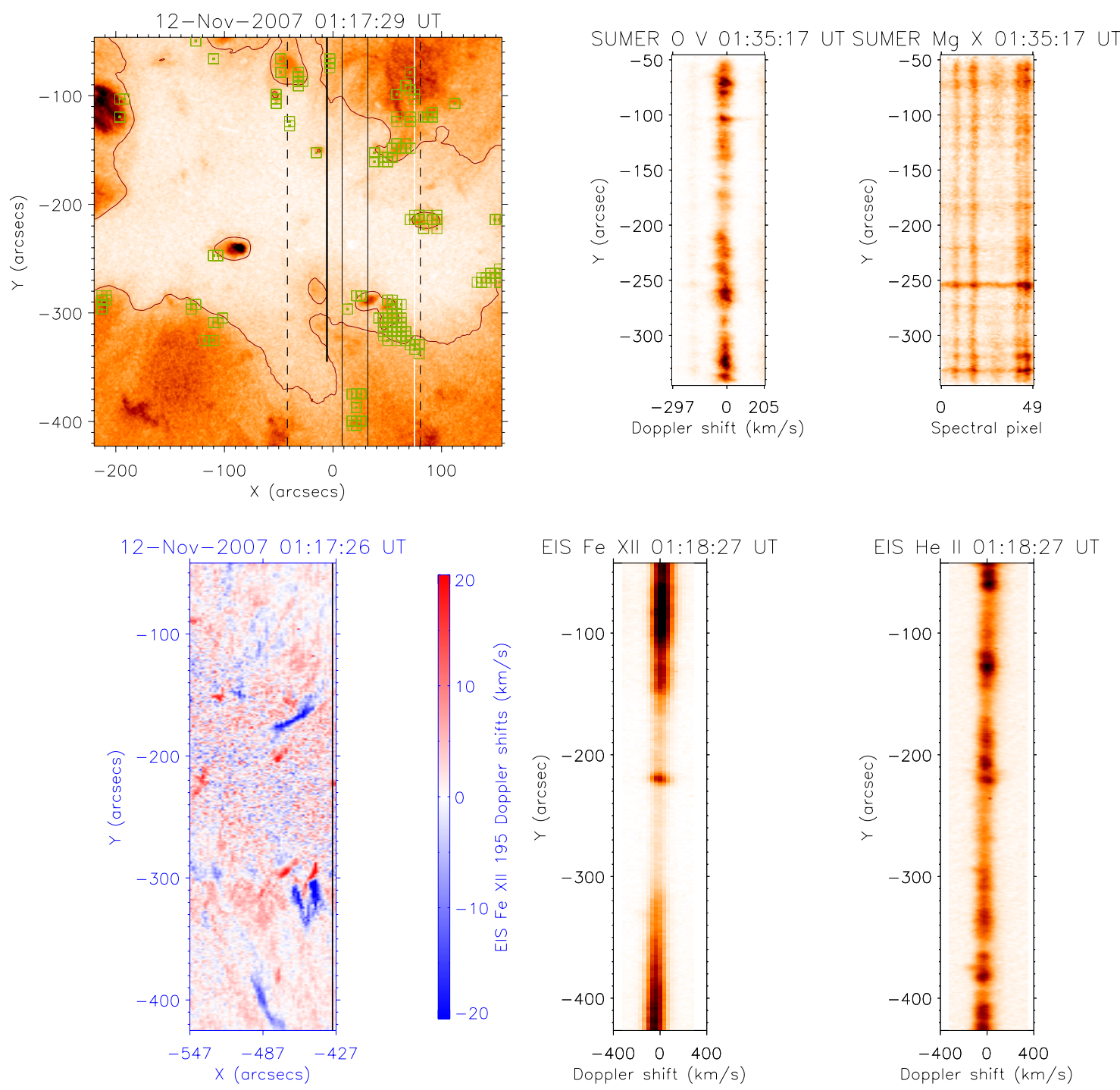

Fig. A.2. Animation of co-temporal images of the coronal hole dataset on November 12 including at the top XRT images with the EIS big raster FOV overplotted with dashed lines, the EIS small raster with solid long lines and the SUMER slit position with solid short line at the given time, and intensity of SUMER O v $1259.54 \AA$ and SUMER Mg x $1249.90 \AA$ windows. The overplotted white line represents the EIS slit position at the given time. Bottom from left to right: EIS Doppler maps, Fe XII $195.12 \AA$, and He II $256.32 \AA$ intensity along the slit at the given time. 